Chapter 4

\title{
Lipid-Based Nanoparticles and Microbubbles - Multifunctional Lipid-Based Biocompatible Particles for in vivo Imaging and Theranostics
}

\author{
Jaroslav Turánek, Andrew D. Miller, \\ Zuzana Kauerová, Róbert Lukáč, Josef Mašek, \\ Štěpán Koudelka and Milan Raška
}

Additional information is available at the end of the chapter

http://dx.doi.org/10.5772/59870

\section{Introduction}

\subsection{Summary}

Lipid-based nanoparticles (LNPs) are both biocompatible and biodegradable so very well suited to medicinal application in targeted drug delivery and in vivo imaging. Microbubbles (MB) are another emerging platform for functional delivery of drugs and in vivo imaging. This chapter is focused on the principles of LNP and MB construction, action and application, concluding with a section on the medical benefits that may result from bringing LNP and MB technologies together. Some applications are mentioned in brief to demonstrate the diagnostic and therapeutic potential of LNPs and MBs. More specific detail on applications is provided elsewhere in this book.

\section{LNP fundamentals}

Structural lipids such as cholesterol and glycerophospholipids are the major components of biological membranes. Without doubt, one of the great triumphs of nature is the manner in which lipid molecules such as these are able to form into cellular membranes sufficient to compartmentalize volumes within cells and between cells. In short, lipids are able to form the vast macromolecular assemblies that come to make up cellular membranes and other barriers 
in nature precisely because they have an unrivalled capability for self-association, driven by weak short range forces and the hydrophobic effect (Figure 1) [1]. This capacity for selfassociation can be exploited in the laboratory in order to create self-assembly LNPs (approx $100 \mathrm{~nm}$ in diameter) (Figure 2) that are proving to be a powerful platform to enable the functional delivery of active pharmaceutical ingredients (APIs) to target cells in vivo. Suitable APIs might include biopharmaceutical agents (e.g. DNA, RNA interference effectors), selected small molecule drugs (e.g. anticancer cytotoxic drugs), and/or imaging agents (e.g. magnetic resonance imaging contrast metals - gadolinium (III) $\left(\mathrm{Gd}^{3+}\right)$, radiometals, and/or fluorescent probes). The number of lipid variations is almost limitless, so too the number and variety of possible LNPs that may be produced for use in biological situations. Therefore, LNPs offer the potential opportunity for tailor-made preparation and production leading in the future even to the possibility of LNP-mediated personalized medicine, no less [2, 3].

a)

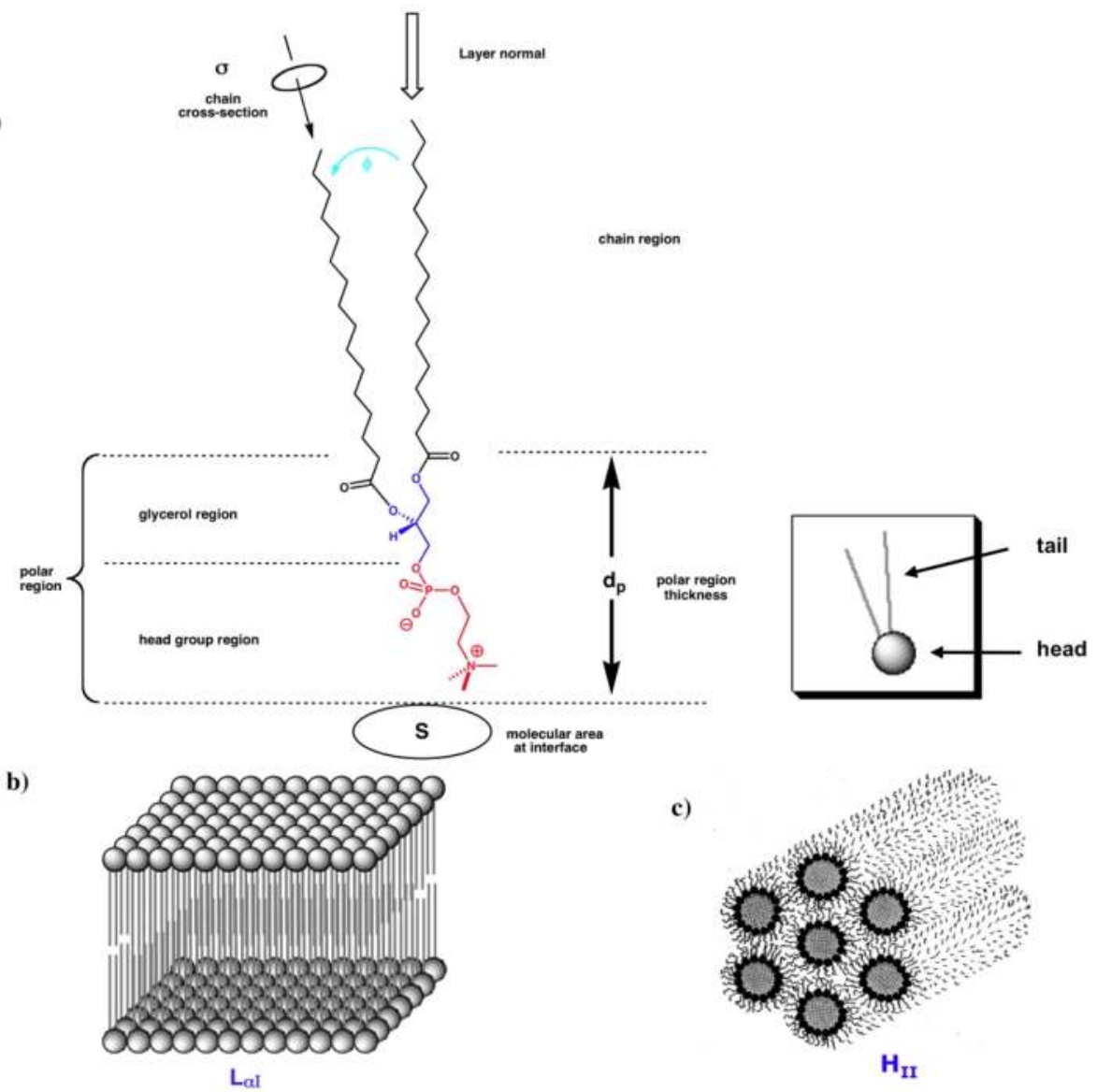

Figure 1. Principles of lipid structure and self-assembly. 
Structural lipids of all types consist broadly of a non-polar, hydrophobic "chain" or "tail" region attached to a "polar" or "head" region (a); biological membranes primarily adopt a normal topology lamellar L $\alpha \mathrm{I}$ fluid mesophase (bilayer) structure (b); under certain circumstances biological membranes adopt other mesophases in particular the inverse topology hexagonal HII fluid mesophase (c), where hydrophobic chain regions face outwards and hydrophilic polar regions face inwards to form aqueous channels (the darker circles). (Diagrams reproduced from [1])

However, and there always is a however, LNPs that have seen service in vivo are turning out to have one fundamental design weakness which can be summarized by saying that those chemical modifications to LNP surfaces that are necessary for such nanoparticles to be stable to storage and in biological fluids, plus minimally visible to a host immune system, now turn out to limit the efficiency of functional delivery once LNPs reach their target cells. Accordingly, one of the primary ways to overcome this problem in recent years has been to introduce the concept of nanoparticle triggerability to LNP design [2, 4]. Nanoparticles possess triggerability (or are said to be triggerable) when designed for stability in biological fluids (from a desired point of administration to disease-target cells) then become triggered for the controlled release of associated APIs at target cells either through local changes in local endogenous (intrinsic) conditions, or through the application of an exogenous (extrinsic) stimulus trained onto target cell regions where nanoparticles are also located. What we are now learning is that MBs used in combination with ultrasound can be used in with LNPs, to provide a potent way to introduce LNP triggerability. Moreover, the introduction of ultrasound critically introduces an opportunity for real-time, diagnostic imaging of LNP mediated delivery of APIs to target cells in vivo. Nanoparticles that combine functional API delivery in vivo with real-time, diagnostic imaging are known as theranostic nanoparticles (TNPs) [5]. Most importantly, TNPs offer the opportunity for true image-guided therapy. As such this may well be a primary future of the MBs-ultrasound-LNP combination as the rest of this chapter will now aim to demonstrate.

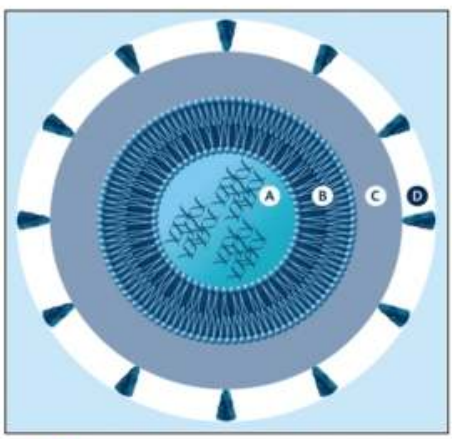

$A B$ systems; in vitro, ex vivo Local/regional use in vivo
A; active pharmaceutical ingredient

-e.g. nucleic acids, drugs, imaging agents

B; compaction/association agents

- lipids or lipid related in this instance

C; stealth/biocompatibility polymer layer

D; biological recognition ligand layer

$A B C D$ nanoparticles constructed

from tool-kits of synthetic chemical components

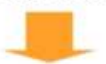

Tailor-made delivery solutions

$A B C / A B C D$ systems; in vivo

Figure 2. Functional lipid-based nanoparticle delivery systems. 
In functional lipid-based nanoparticles (LNPs), active pharmaceutical ingredients (APIs) (A) are condensed within functional concentric layers of chemical components designed for delivery into cells and intracellular trafficking (B components, primarily lipids and lipidrelated components), biological stability (C stealth/biocompatibility components-typically Polyethylene Glycol [PEG]) and biological targeting to target cells (D components, biological receptor-specific targeting ligands) [2,3].

\section{Liposomes to LNPs}

Liposomes are the progenitors of all LNPs, and they are formulated directly from lipid constituents (Figure 3). Liposomes can be prepared by various techniques. The method based on hydration of lipid film represents the most exploited technique for preparation of liposomes. The lipids of known molarities are dissolved in organic solution which is subsequently slowly evaporated in vacuo to produce a thin film. The film is hydrated with a suitable aqueous buffer at temperature, which is generally above transition temperature of lipids used. Aqueous buffer contains also compound which are to be entrapped into liposomes. Size of liposomes can be reduced by freeze-thaw procedure followed by sonication, extrusion, microfluidisation or high pressure homogenisation. These procedures can tune size distribution of final liposomal preparation to desired values. The main parameters characterising liposomes are their size, spherical shape and degree of lamellarity. Liposomes may consist of one bilayer (unilamellar), several bilayers (oligolamellar) or multiple bilayers (multilamellar). Membrane rigidity can be adjusted using appropriate lipids and membrane fluidity may be controlled with the use of phospholipids with higher or lower transition temperature.
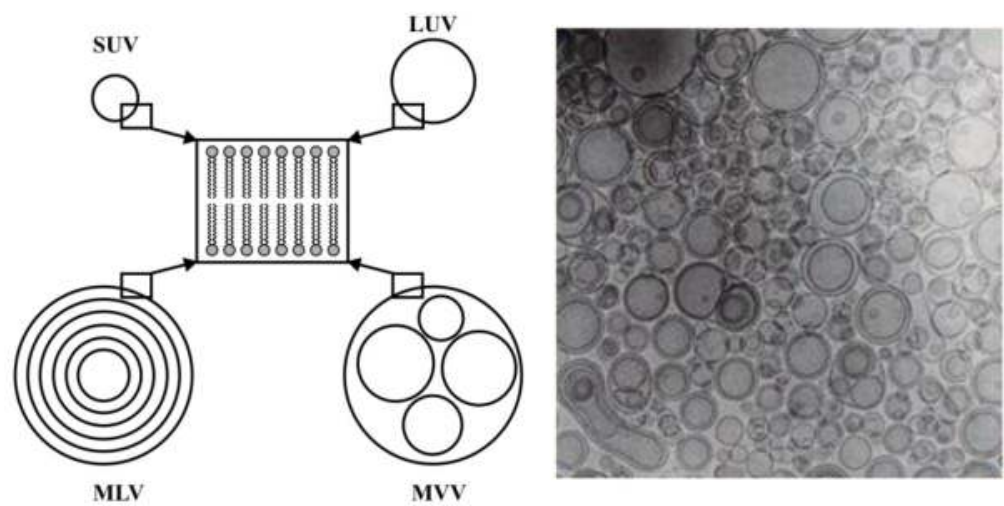

Figure 3. Various morphological types of liposomes and real appearance in cryoelectron microscopy.

Small unilamellar vesicles (SUV); Large unilamellar vesicles (LUV); Multilamellar vesicles concentric (MLV); Multivesicular vesicles (MVV) 
Liposomes are generally formulated on the nm scale and can be further size refined by passage through physical membrane pores of known size (extrusion). Liposomes are typically characterised by their size, spherical shape and degree of lamellarity. They may be composed of a single bilayer (unilamellar), a few bilayers (oligolamellar), or multiple bilayers (multilamellar). The rigidity of the membrane can also be modified with the use of suitable lipids; and the fluidity of the membrane may be controlled using phospholipids with higher or lower $\mathrm{L}_{\alpha \mathrm{I}}-$ $\mathrm{H}_{\text {II }}$ mesophase transition temperatures. In general lipids of stearic acids (fully saturated C18 hydrocarbon chains) bestow rigidity to liposome structures (by favouring $\mathrm{L}_{\alpha \mathrm{I}}$ fluid mesophases), whilst lipids of oleic acid ( $\Delta 9$ unsaturated $C 18$ hydrophobic chains) can result in less stable structures (by favouring $\mathrm{H}_{\mathrm{II}}$ fluid mesophases). Liposomes have traditionally been used as some of the very earliest nanodrug delivery vehicles, encapsulating water-soluble drugs within their central aqueous cavities in order to improve drug pharmacokinetics [6-8]. Liposome spherical diameters can be investigated by using scattered light off the surface of the particles with photon correlation spectroscopy. Alternatively cryo-TEM can be used to provide visual images of liposome sizes and shape. The surface charge of liposomes can be obtained by measuring their zeta potential (electrokinetic potential). Liposome associated techniques and characteristics have all been employed in more recent time times to prepare more chemically diverse LNPs.

\section{Cellular imaging}

Cellular imaging is "the visualisation of specific cells in an intact animal". This term also collectively denotes the visualisation of any type of the entire cell under different conditions [9]. While cellular imaging applies to the imaging of whole cells, molecular imaging deals with the visualisation of molecules and sub-cellular components within individual cells [10]. The exploitation of molecular probes or imaging agents is essential for the use of both cellular and molecular imaging strategies. Despite the fact that the method of cellular imaging of extrinsic fluorophores is highly efficient, the depth of tissue from which the signal emitted represents often a limiting factor for this technique. The possible use of positron emission tomography (PET) or single-photon computed tomography (SPECT) methods is also limited due to shortlived radioisotopes and poor spatial resolution. However, three-dimensional images of tissues containing water can be obtained by magnetic resonance imaging (MRI). This technique is characterised by high levels of spatial resolution and imaging of depth tissues is achieved.

A primary limitation of MRI is an inherent lack of sensitivity that can be overcome through the use of contrast agents that enhance signal sensitivity and hence image quality. MRI contrast agents consist of molecules that incorporate a paramagnetic metal ion, most commonly gadolinium (III) $\left(\mathrm{Gd}^{3+}\right)$ or Iron $\left(\mathrm{Fe}^{3+} / \mathrm{Fe}^{2+}\right)$. The improvement in image quality derives from the modulating effects of the coordinated metal ions on longitudinal $\left(T_{1}\right)$ or transverse $\left(T_{2}\right)$ relaxation times associated with proton resonance signals emanating from bulk water molecules surrounding the coordinated metal ions. Even though contrast agents incorporating Gd3increase both 1//T1 and 1/T2 relaxivities, they are commonly used for T1-weighed (positive bright) contrast imaging, on condition that $1 / \mathrm{T} 1$ contribution is higher in tissue than the 
corresponding effect on 1/T2 enhancement. Due to the fact that more considerable increases in 1/T2 are induced by iron containing contrast agents, they are usually used in T2- weighed (negative dark) contrast imaging [11]. Despite the most frequently used MRI contrast agents are thermodynamically and kinetically stable low molecular weight $\mathrm{Gd}^{3+}$ complexes (that promote MRI contrast by non-specific enhancement of water proton relaxation rates within the blood pool), there has been a realization of the need for imaging LNPs suitable for the targeted delivery of imaging agents to sites of disease pathology such as cancerous lesions in order to obtain clear contrast images of their extent and location.

For cell populations to be imaged by MRI in vivo, these populations must be distinguishable from natural background noise signal. For this to happen, cells of interest should become labelled with appropriate agents, making use of controlling mechanisms such as enhanced permeability and retention (EPR) mechanism for imaging agents to reach target cells $[12,13,14]$, followed by controlled target cell entry. According to Frangioni et al., the ideal cellular label should have the following features: a strong signalling effect, good biocompatibility, does not interfere with cellular genetics, remains retained only in the target cell population, and allows temporal imaging for long-lasting periods [15]. Controlled target cell entry is preferably by ligand-specific receptor-mediated cell entry processes, although alternative mechanisms of cell entry may also be acceptable (Figure 4) [16].

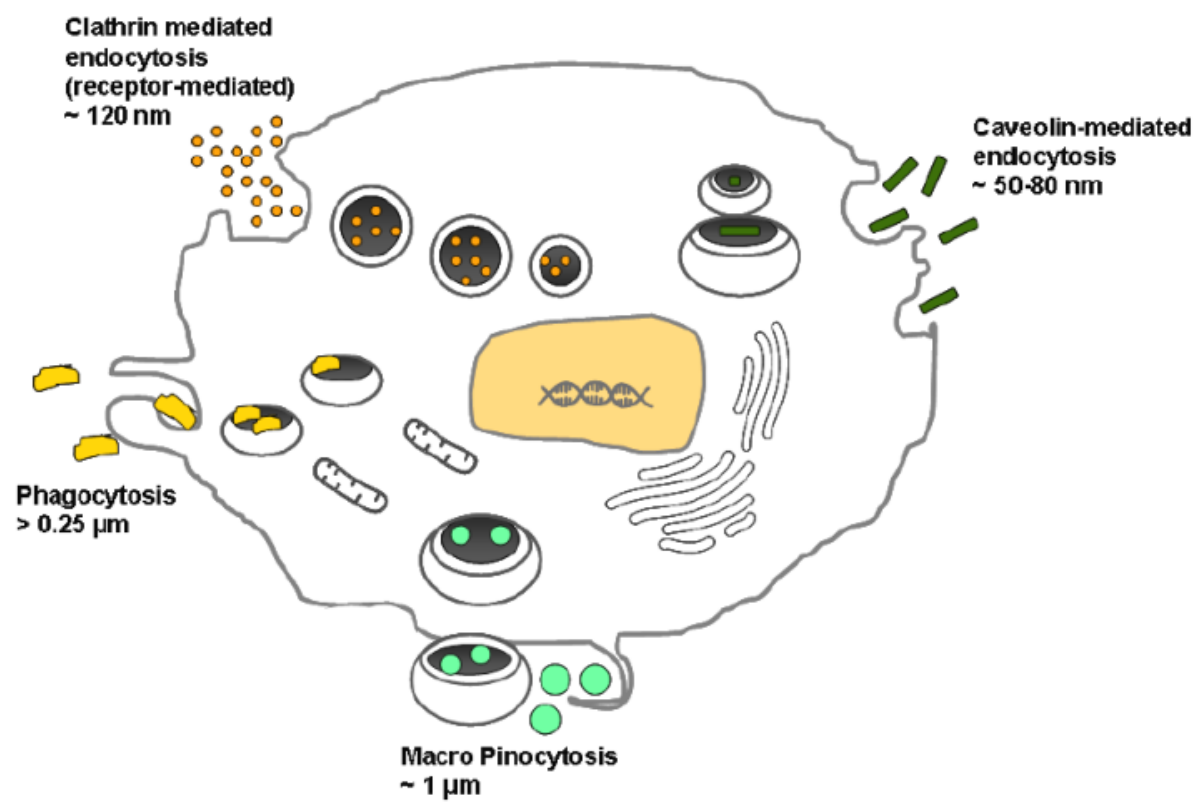

Figure 4. Cell entry mechanisms: the various uptake mechanisms of macromolecules and nanoparticles into cells are indicated according to their size (Adapted from [14]). 
LNPs are an ideal platform to combine multimodal imaging (e.g. MRI contrast agent and fluorescent probes) with multiple functionalities in order to effect multimodal cellular labelling. Targeting of LNPs to various tissues and cells (e.g. tumour) can be accomplished by conjugation of LNP surfaces with biological receptor-specific targeting ligands (e.g. monoclonal antibodies, folate, RGD peptides) [2,3]. One of the best examples of such an imaging LNP recently described in the literature is shown (Figure 5). This imaging LNP system (Gadonano$\mathrm{F}$ ) is a novel $\mathrm{Gd}^{3+}$-containing, imaging LNP system that was designed to act as a folate receptor (FR)-targeted, positive contrast agent to enable magnetic resonance imaging (MRI) of FRpresenting cancerous lesions (primary and metastatic) including breast, lung, intestinal, renal, and ovarian cancers.

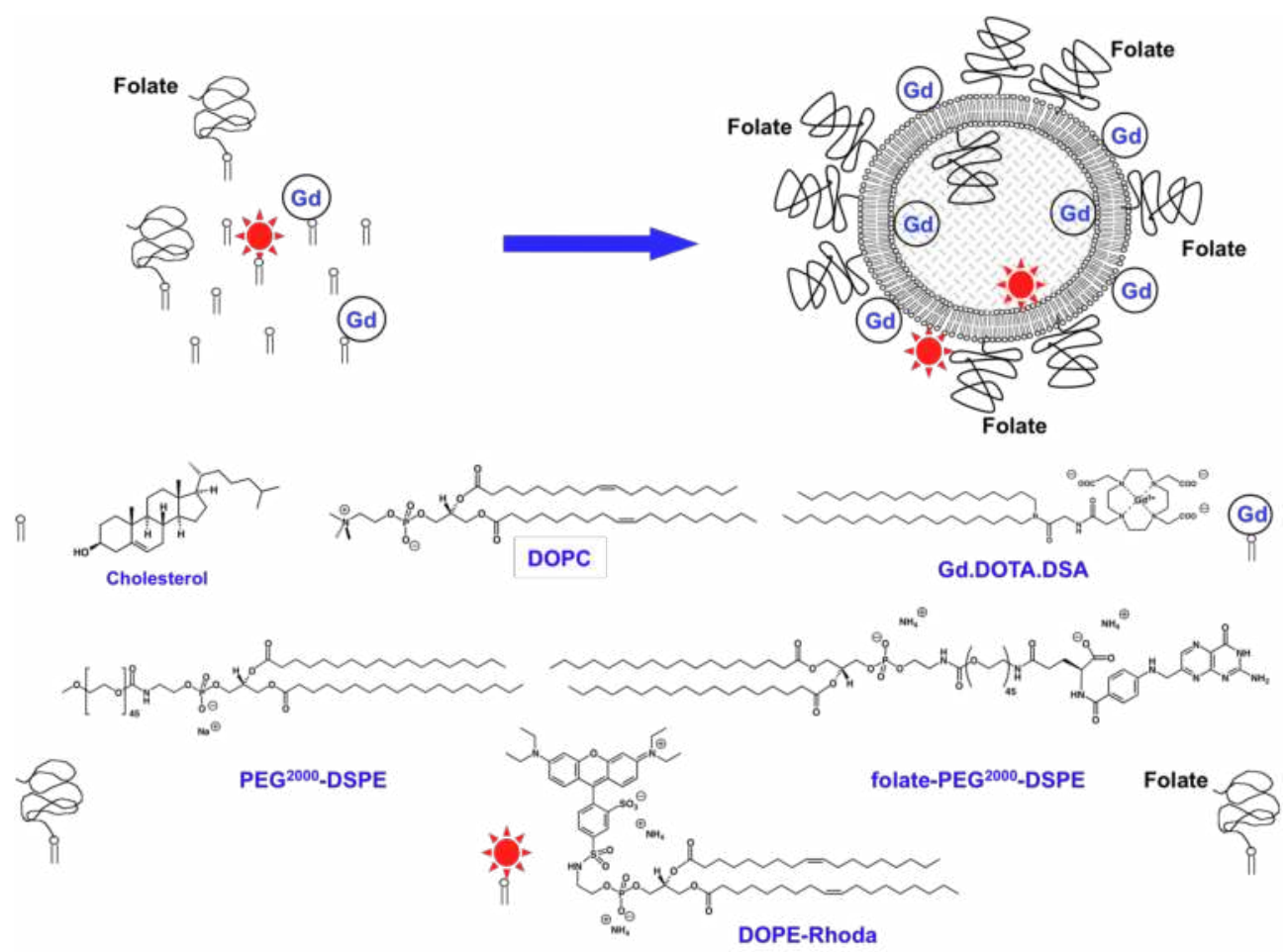

Figure 5. Multimodal imaging Gadonano-F LNPs for targeted delivery of MRI contrast agents to tumour cells.

Gadonano-F LNPs are prepared as above with the indicated lipid components. Double labelling is made possible using gadolinium metallochelating lipid (Gd.DOTA.DSA) for MRI positive contrast imaging and rhodamine fluorescent probe conjugated lipid (DOPE-Rhoda) for fluorescence imaging. Folate association with the Gadonano-F LNP surfaces is made possible using a polyethylene glycol (PEG) lipid (folate-PEG ${ }^{2000}-\mathrm{DSPE}$ ), ensuring that Gadonano-F LNPs are enabled for FR-specific targeting to tumour cells in vivo. The remaining PEG lipid ( $\mathrm{PEG}^{2000}$-DSPE) ensures that the Gadonano-F LNPs possess stability in biological fluids (e.g. serum) (see [14]). 
There are a number of pathways by which LNPs could enter mammalian cells, namely by phagocytosis, pinocytosis, clathrin and calveolin independent endocytosis [9, 17]. Of these, phagocytosis is a process of engulfing and internalisation of large particles ( $1 \mathrm{um}$ ) by cells. The majority of phagocytic cells of the immune system, including macrophages, neutrophils, monocytes, and microglia, are able to internalise foreign bodies via phagocytosis. Pinocytosis is a method of particle internalisation by non-phagocytic cells but this is a very non-specific mechanism of internalisation into cells [9]. On the other hand, clathrin-mediated endocytosis (receptor-mediated) and caveolin-mediated endocytosis are much the more specific means of cellular entry, of which the first is ideal for entry of LNPs to cells.

In original preclinical laboratory experiments, Gadonano-F LNPs were prepared and used to image xenograft tumours (IGROV-1) in mice. At just 2h post-injection the Gadonano-F LNPs mediated a similar signal enhancement to that attained at $24 \mathrm{~h}$ post-injection by control (Gadonano LNPs, lacking folate ligand) (Figure 6). Specifically, Gadonano LNPs accumulated in tumour by the EPR mechanism ("passive" targeting) [12,13], and labelled cells for MRI over a period of $24 \mathrm{~h}$. These imaging LNPs were seen to access the entire hyperpermeable, living volume of the tumour while being excluded from the necrotic regions. By contrast, the FRtargeted Gadonano-F LNPs accumulated in the tumour and labelled cells for MRI from $2 \mathrm{~h}$ onwards, owing to EPR mediated tumour uptake from the blood pool followed by rapid FRmediated cell entry probably involving clathrin-mediated endocytosis. FR-targeted imaging LNPs accumulated in more perivascular and macrophage-rich locations [14,18].
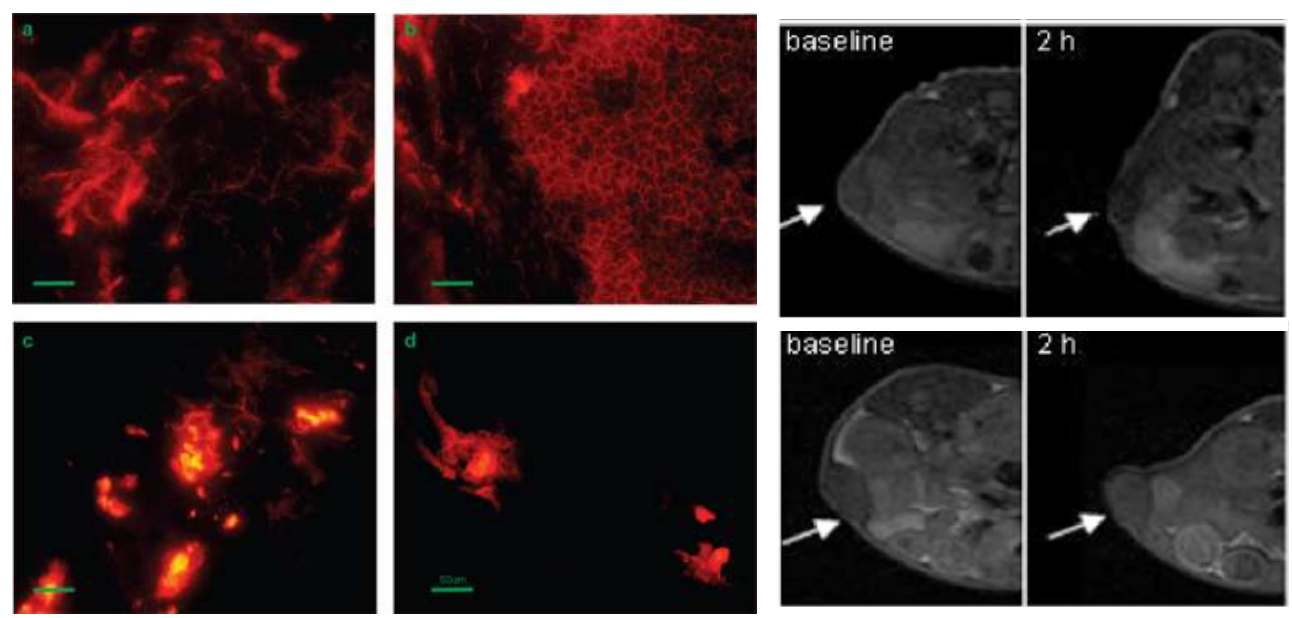

Figure 6. Fluorescence microscopy and MRI contrast imaging of $24 \mathrm{~h}$ post imaging LNP injection.

On the left $24 \mathrm{~h}$ post imaging LNP administration, (a) surface IGROV-1 tumor slices, Gadonano LNPs, (b) midtumor IGROV-1 slices, Gadonano LNPs, (c) surface IGROV-1 tumor slices, Gadonano-F LNPs, and (d) IGROV-1 midtumor slices, Gadonano-F LNPs (green bar represents $50 \mu \mathrm{m}$ ). On the right, MRI contrast images of IGROV-1 tumour bearing Balb/C nude mice: 
images are from control mice injected with Gadonano LNPs (top); images are of mice injected with Gadonano-F LNPs (bottom) (white arrow shows location of tumour) (Adapted from [18])

Rapid and effective imaging of FR-bearing tumours by Gadonano-F LNPs in preclinical experiments, has since led to these LNPs being prepared for clinical trial by GlobalAcorn Ltd under the name GA101 NANO-GdF (Gadonano LNPs are also being developed for clinical trial by GlobalAcorn Ltd under the name GA100 NANO-Gd). Rapid and specific imaging of diseased tissues and tumours will be of paramount importance for preclinical research and also future diagnostic and theranostic applications in clinic [2, 3].

\section{Microbubbles (MBs)}

Since the MBs were first proposed as suitable tools for ultrasound contrast agents more than three decades ago, there has been a remarkable progress in the field. In accordance with that, the research has been particularly concerned with the MB preparation techniques and their standardization, in order to ensure a steady composition and size distribution of MBs, both representing crucial features for the stability of MBs in vivo and thus providing a sufficient backscatter for ultrasound imaging. Although the very first MBs were generally considered to contribute to improvement of the imaging techniques, they were soon proved to serve as excellent drug and gene delivery systems both in diagnostics and therapy. Much has been discussed on ultrasound molecular imaging using MBs as contrast agents, including novel preparation techniques and their general design; however, the choice of a convenient system for drug and gene delivery and concept of MB functionalization is still an issue worth consideration. In this chapter, potential ways for application of MBs as contrast agents together with drug and gene delivery are shown, relating to the recently used functionalized MBs in vitro and in vivo.

MBs are small gas-filled microspheres whose size typically ranges between 1 and $10 \mu \mathrm{m}$ in diameter see (Figure 7). They comprise a gas-filled core, usually formed by inert, high molecular gases such as sulphur hexafluoride or perfluorocarbons, which due to their decreased solubility and low diffusion prolong the lifespan of MBs in circulation [19], by means of a stabilizing shell of lipid, polymer and/or protein $[20,21]$. The composition of the shell determines the stiffness and therefore the stability of MBs in blood flow, where instability is caused by exposure of MBs to the rigors of systemic circulation [19, 22]. Limits on stability can affect the scope of ultrasound imaging utilization in vivo since the half-life of MBs depends on their stability. Generally, lipid shelled MBs are considered thinner and more flexible, whilst polymer and protein-shelled bubbles have a thicker and more rigid shell [23]. A thick permeable shell is beneficial to enhance the MB stability (also reducing the gas diffusion) and thus might be used for ultrasound drug delivery purposes [19, 24, 25]. However, the shell ought to only slightly limit the vibration of $\mathrm{MBs}$ in the ultrasound field generated by echo imaging systems (otherwise they do not provide sufficient contrast).

Another important aspect for any $\mathrm{MB}$ formulation is their size distribution that dictates the acoustic properties of MBs [22]. As the MBs smaller than $1 \mu \mathrm{m}$ in diameter produce minimal 
acoustic contrast, the intent is to produce larger MBs [26-29]. On the other hand, the MB size distribution should not exceed $10 \mu \mathrm{m}$ of size in diameter; otherwise they get quickly filtered by the lungs and can pose a risk of embolus $[22,30]$. Furthermore, the size distribution is also believed to affect the drug delivery $[22,31,32]$.

\section{Ultrasound imaging}

In the field of molecular imaging, ultrasound represents a unique imaging technique as it provides real-time images in a non-invasive way using relatively cheap and easily portable equipment; thus, ultrasonography is considered to be currently most widely used diagnostic imaging modality [19]. However, since the liquid phase material with low compressibility, such as blood, scatters the sound waves poorly, contrast agents were proposed to improve the ultrasound images, offering the following general advantages: a very small intravenous injection is needed for imaging; and they are composed of non-toxic, biodegradable materials [30]. There are several types of ultrasound contrast agents, such as liquid emulsions, liposomes, and gas-filled MBs [33]. MBs will be described in detail in this chapter. Since the MB-based contrast agents are similar in size and shape to erythrocytes and also rheologically behave similarly to the blood, they appear to be of utmost convenience for ultrasound imaging systems, providing bright contrast [30]. Moreover, due to their larger size (compared to lipid and polymer based nanocarriers), systematically delivered MBs do not extravasate; instead they remain in circulation until they dissolve or are actively cleared by the mononuclear phagocyte system (MPS) [21, 34, 35].

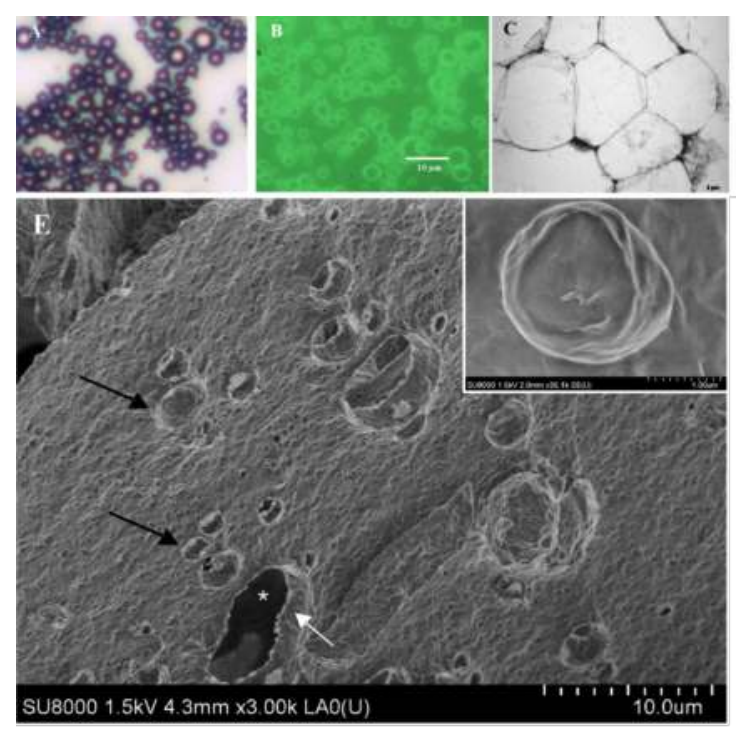

Figure 7. Pictures of MB obtained by various imaging techniques. 
a. Light microscopy of DPPC/ DPPC/1\% DOGS-NTA-Ni MB with carboxyfluorescein-PE $(1 \%)$

b. The same MBs as in (A) observed by epifluorescence microscopy.

c. TEM of MBs. During desiccation of the sample in the vacuum interior of electron microscope, the MB are pressed to each other, which results in the deformed polygonal shape

d. SEM picture of lyophilised MBs. Intact MB (black arrows), crushed MB shell (white arrow), hollow inside of MB (white asterisk). Insert: detail of the surface of lyophilised MB.

(Adapted from [48])

\section{MBs in the ultrasound field - Different aspects}

When exposed to low acoustic pressures, MBs tend to oscillate in a symmetrical, linear way [36], which means that their expansion and compression is inversely proportional to the local ultrasound pressure [37]; the phenomenon is known as a stable cavitation or non-inertial cavitation [38] and comprises stable, low amplitude oscillations of MBs (Figure 8) [23]. Such stable oscillations create a liquid flow around the MBs, the so called microstreams, which can apply a shear stress on cell membranes resulting in a transient opening of the cell membranes, as the shear stress related to micro streaming is relatively high compared to the shear stress associated with blood flow $[23,36,39]$. With the driving pressure turning into violent or inertial cavitation [38, 40], MBs undergo rapid expansion and contraction, which result in a violent bubble destruction [21]. The collapse of MBs is accompanied by shock waves generated in the fluid near the MB; furthermore, jet formation may occur, which can both contribute to a cell membrane perforation and increased permeabilization of blood vessels, thus improving extravasation of circulating drugs into target cells and tissues [20, 23, 41-44]. This phenomenon is called sonoporation [45] and has been proved visualisation of the pores by scanning electron microscopy, showing the pores in the plasma membrane (Figure 9) [46, 47]. Despite initial belief that inertial cavitation of MBs was required to enhance uptake of low molecular weight drugs, there is now mounting evidence that these stable oscillations may also stimulate the intracellular delivery of macromolecular drugs and nanoparticles [36]. Two mechanisms have been postulated to contribute to the uptake of cell impermeable molecules: the formation of small pores and endocytosis [46, 49]. Whilst the involvement of endocytosis was shown to be more extensive for larger molecules, the pore formation is considered the main mechanism for low-weight molecules, such as dextrans [49]. Moreover, when studied in a single-cell model, it was reported that a direct contact is needed to induce pore formation by stable cavitation, as larger distances would hamper direct mechanical cavitating $\mathrm{MB}$ and cell membrane and decrease the influence of microstreaming on the cell membrane (Figure 10) [36, 50-52]. Additionally, MBs facilitate cavitation related phenomena, such as temperature rise and free radical formation $[21,42,53]$; it is assumed that the free radicals increase the cell membrane permeability for $\mathrm{Ca}^{2+}$ in primary endothelial cells and cardiomyocytes [54-56]. 
Last but not least, acoustic radiation forces that may translate MBs in the direction of the propagating ultrasound wave should be taken into account $[21,36,57,58]$. Not only can the acoustic radiation forces be applied to adhere to a specific target, but they can also help to push the MBs towards the cell surface and thus stimulate interaction with the cell membrane and promote drug delivery to specific cells [59-62]. Through acoustic radiation forces, MBs can squeeze through endothelium and tunnel through soft tissue, possibly increasing the deposition of shell-loaded materials beyond the vasculature [21, 63, 64].

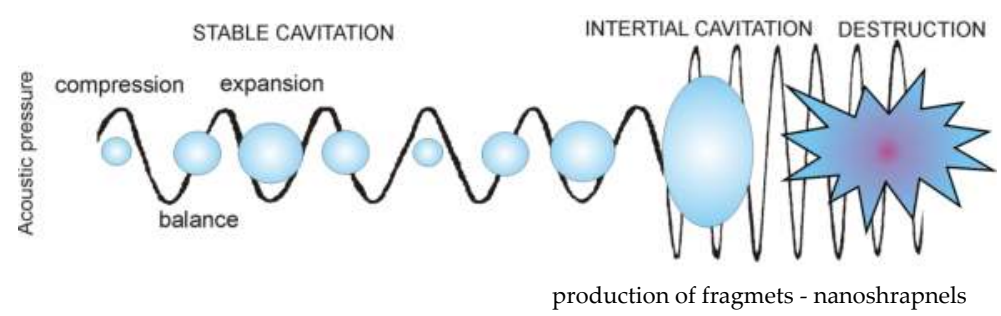

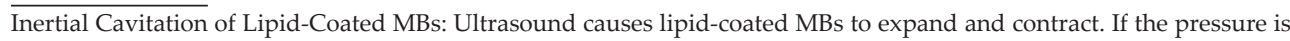
below a critical value, called the inertial cavitation threshold, then the bubble undergoes sustained oscillations - stable cavitation. If the pressure is above the inertial cavitation threshold, then inertial forces govern the collapse; this is typically associated with the bubble imploding and breaking into small fragments - nanoshrapnels. The inertial cavitation threshold pressure depends on the material properties of the lipid coating (e.g., area expansion modulus and surface tension), which are set by the monolayer composition (lipid chain length, PEG coating, nanoparticle bound to the MB surface)

Figure 8. Effect of acoustic pressure on MB oscillation and explosion.

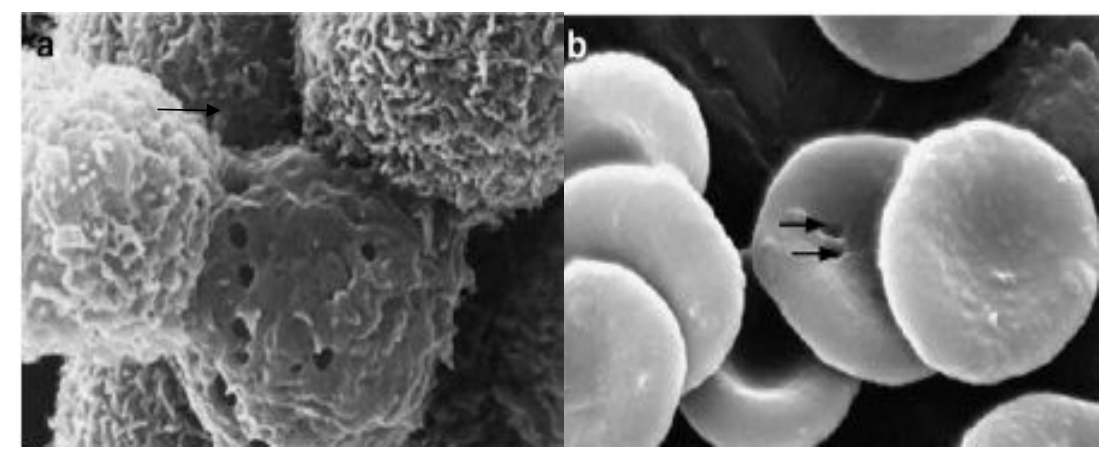

$\overline{\text { GSEM micrograph }}$ of cells after sonoporation and fixation. The cells were insonated with $2.25 \mathrm{MHz}$ and $570 \mathrm{kPa}$ peak negative pressure pulses in the presence of MBs. (a) MAT B III cells; (b) red blood cells. Adapted from [45]. Arrows indicate pores in the cell membrane. (http://amazingbubbleworld.blogspot.cz/2012/05/classifications-of-cavitation.html)

Figure 9. MBs assisted sonoporation of cells. 

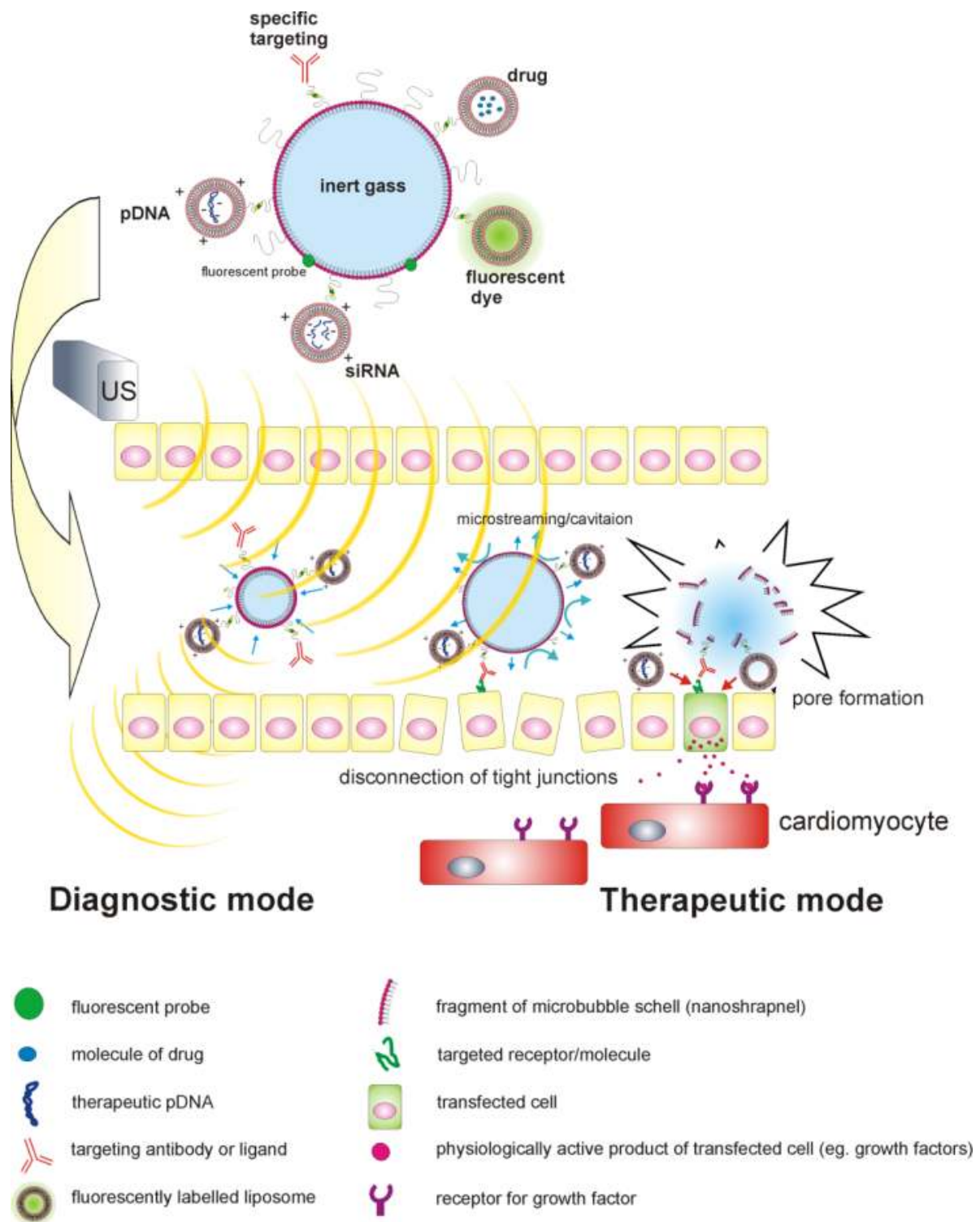

Figure 10. Various aspect of haw MBs can effect endothelial cell in capillary vessels. 


\section{Commercial preparations}

The use of MBs in echocardiography was first reported in 1968 [65]. Since that time great progress has been done in preparation of MBs for human and veterinary use. The following three commercial MB agents Optison TM (GE Healthcare, WI, USA), Definity ${ }^{\circledR}$ (Lantheus Medical Imaging, MA, USA) and SonoVue ${ }^{\circledR}$ (Bracco, Milano, Italy) currently hold the license for clinical diagnostic applications (Table 1). The size of the commercial MBs in these agents is usually larger than $1 \mathrm{um}$ with imaging durations being 5-10 min.

\begin{tabular}{ccccccc}
\hline MB type & Manufacturer & Shell material & Gas & $\begin{array}{c}\text { Mean size } \\
(\mu \mathrm{m})\end{array}$ & $\begin{array}{c}\text { Concentration } \\
(\mathbf{M B} / \mathbf{m L})\end{array}$ & $\begin{array}{c}\text { MB half life } \\
(\mathbf{m i n})\end{array}$ \\
\hline Optison $^{\mathrm{TM}}$ & GE Healthcare & Albumin & $\mathrm{C}_{3} \mathrm{~F}_{8}$ & $2.0-4.5$ & $5-8 \times 10^{8}$ & $2.5-4.5$ \\
Definity ${ }^{\circledR}$ & $\begin{array}{c}\text { Lantheus Medical } \\
\text { Imaging }\end{array}$ & Phospholipid & $\mathrm{C}_{3} \mathrm{~F}_{8}$ & $1.1-3.3$ & $1.2 \times 10^{10}$ & $2-10$ \\
& Bracco & Phospholipid & $\mathrm{CF}_{6}$ & $2.0-8.0$ & $0.9-6 \times 10^{10}$ & $3-6$ \\
\hline
\end{tabular}

Table 1. Specifications of commercial and self-made MBs.

\section{Multifunctional MBs for ultrasound imaging and theranostics}

The concept of therapeutic agents being encapsulated in or conjugated with MBs has been developed over the past few years. In addition to the synergistic effects of ultrasound and MBs to enhance the permeability of biological barriers such as cell membranes, small blood vessels, and the BBB, as discussed above, MBs can serve as protective drug carriers. Drugs can be preincorporated into carriers such as liposomes, micelles, or microspheres, and these structures can then be easily attached to lipid MBs, usually via avidin-biotin interactions (Figure 11) [66]. Such advanced MB complexes have extremely high drug loading capacities and the advantage of being able to encapsulate both hydrophilic and hydrophobic drugs. Encapsulating unstable agents protects them from degradation in blood, thus prolonging their half-lives in vivo, improving treatment efficacy, and lowering the required dose [67]. Another advantage is that the encapsulated agents can be released during the ultrasound-triggered MB destruction process. Chemotherapeutic drugs can thus be directly and specifically delivered to target tissues via ultrasound-mediated perforations, whereas the uptake of the drugs by non-target tissues is reduced. The encapsulated agents are conjugated close to the shell of MBs, increasing the opportunity for microstreams, shock waves, and microjets to drive them toward the tissues and enhance their uptake in the ultrasound-treated region [68]. Since MBs act as ultrasound contrast agents, the drug delivery process can also be concurrently monitored by detecting the drug-loaded MBs [69]. 

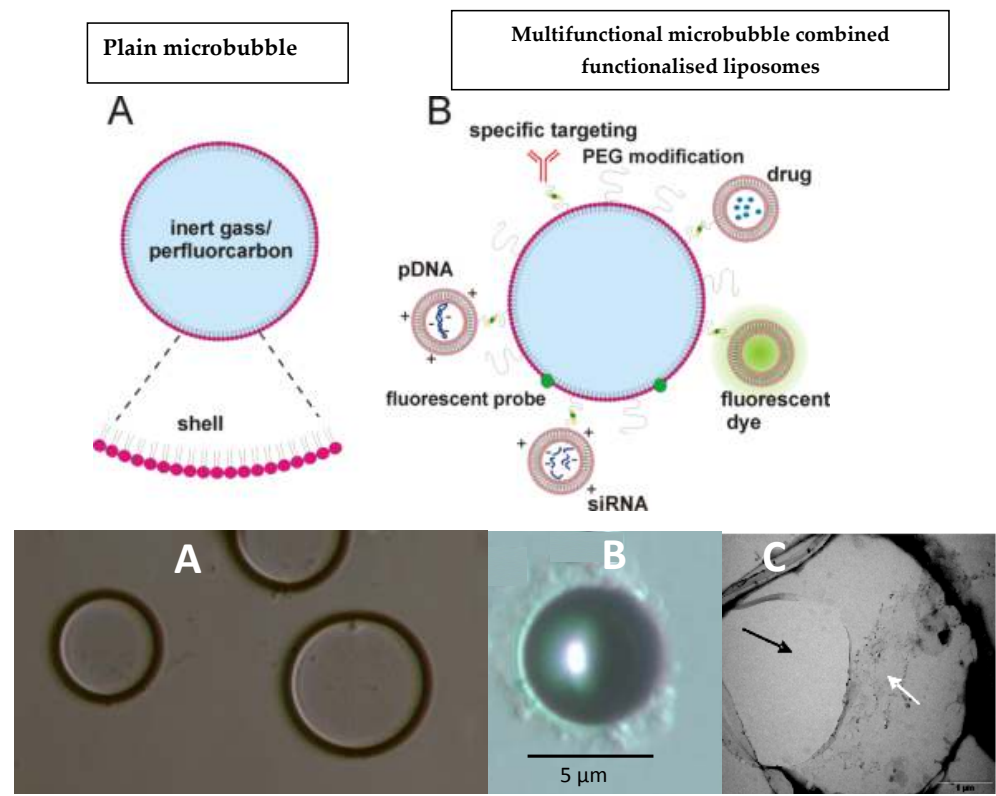

A) Structure of plain MB (upper - schematic picture; bottom - optical microscopy -Nomarski contrast)

B) Functionalised MB with bound liposomes (upper - schematic picture showing various possibility of functionalization and modification; bottom - MB with surface bound liposomes - optical microscopy -Nomarski contrast)

C) Transmission electron microscopy of the functionalised MB with bound liposomes. Black arrow - rupture of MB shell owing to vacuum in electron microscope; white arrows - liposomes bound onto the surface of MBs. (Adapted from [48])

Figure 11. Targeted and multifunctional MBs.

\section{Targeted MBs}

Despite the possibility of employing MBs for targeted ultrasound imaging and drug and gene delivery using the local ultrasound insonation, attaching targeting ligands on the surface of MBs, which leads to their accumulation in the target region (Figure 12), makes the MBs even more specific so that the range of clinical use of MBs is vastly expanded [23]. Owing to their size, MBs are not supposed to leave the vasculature; thus their use is limited to thrombus, endothelial cells, atherosclerotic plaques, blood clots, and organs such as spleen and liver [23, 47]. Recently there have also appeared papers referring to the use of MB-enhanced ultrasound for facilitated brain delivery [70,71]; although the exact mechanism is still unknown, the interaction between capillary walls and acoustically driven MBs was found to be one of the key factors which leads to the disruption of the blood-brain barrier - the main obstacle in the treatment of neurodegenerative diseases [70, 72]. Since the method is non-invasive, according to the authors, it could be the way for treatment of such diseases as Parkinson's disease or Alzheimer's disease. 


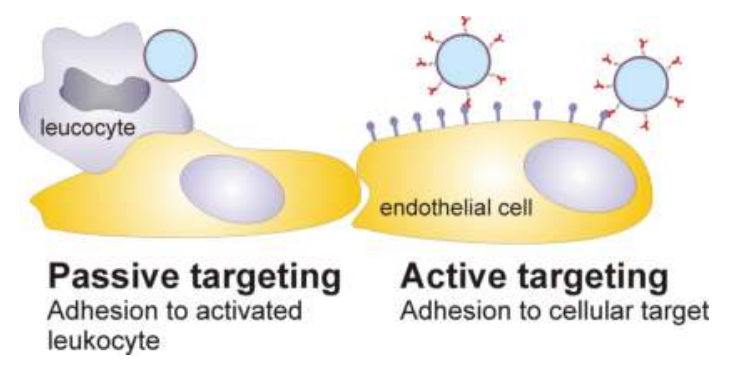

Figure 12. Passive and active targeting of MBs.

There are different ways how to attach the targeting ligands to the shell of MBs that are generally distinguished by the method of preparation. The targeting ligand can be either co-incorporated in the shell during the MB preparation, or attached to the surface of pre-formulated MBs by covalent or non-covalent methods [73, 74]. The simplest targeted MB design is based on the use of a phospholipid, phosphatidylserine, which is incorporated in the MB shell during preparation. Due to the fact that phosphatidylserine is a membrane marker of apoptosis, it promotes the phagocytic uptake of cells and liposomes by Kupffer cells and macrophages [74-76]. While circulating in the bloodstream, MBs containing phosphatidylserine are actively targeting and captured by the Kupffer cells in the liver, macrophages in the spleen and active neutrophils in the areas of inflammation, which is the reason why phosphatidylserine-shell agents are used to detect the foci that lack active phagocytic capacity, e.g. tumour nods in the liver [73, 77-79]. In these agents, the shell is the ligand (phosphatidylserine); since the molecules of phopshatidylserine are kept together by the hydrophobic, van der Waals, and other intermolecular interactions, the ligand is attached in a non-covalent manner [73].

Nevertheless, though prepared by straightforward shear-mixing in one simplestep, this method is convenient only for stable ligands, which are able to survive the extreme conditions during the MB preparations [23]. Particularly some proteins and enzymes might be denatured in the harsh conditions and might lose binding affinity [73]. Therefore a different approach has been applied, involving attachment of ligands after MB preparation: non-covalent (strept)avidinbiotin interaction and covalent binding $[23,73,80,81]$. Considering the fact that the (strept)avidin-biotin interaction has the strongest effective dissociation constant, it has been the most widely used technique in biotechnology [82]. Biotin residues might be attached in one single step to antibodies, carbohydrates and many other targeting ligands, including the MB shell components $[73,83]$. Biotinylated antibodies are abundant and can be easily linked to premanufactured biotinylated bubbles via a streptavidin linker [84]. Nonetheless, this coupling scheme is useful only in preclinical research: streptavidin as a foreign protein is a possible immunogen which might lead to undesired immune response after multiple injections [85].

Another attractive non-covalent strategy is to use the interaction of a metallochelating lipid complex, such as nickel-nitrilotriacetyl lipid, with a multiplicity of histidine residues (His-tag) of a protein [48]. The method offers an easy way to attach a genetically engineered protein to the MB surface with a His-tag, a commonly applied insert in recombinant protein preparation. 
The advantage of metallochelating complex over protein-ligand complexes, e.g. (streptavidin - biotin, glutathion - glutathion transferase) consists in its specific binding and very low immunogenicity, if any [86]. However, except for studies using metallochelating liposomes $[86,87]$, very little research has been devoted to this issue in terms of MBs; therefore there still appears to be a high potential for the His-tag - MB applications in the foreseeable future.

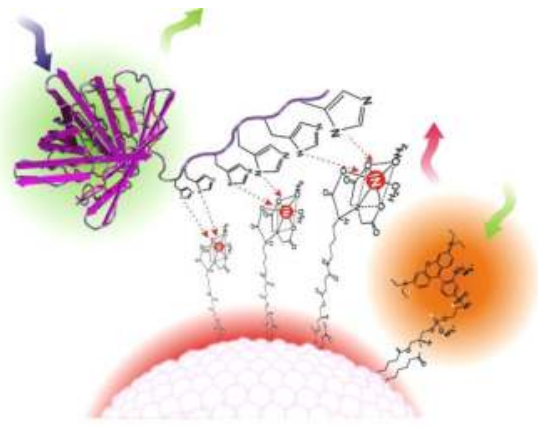

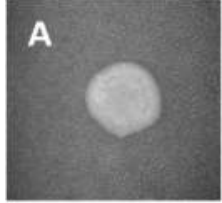
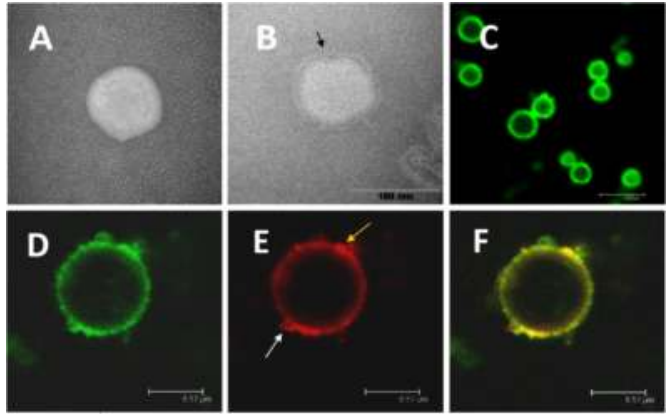

G

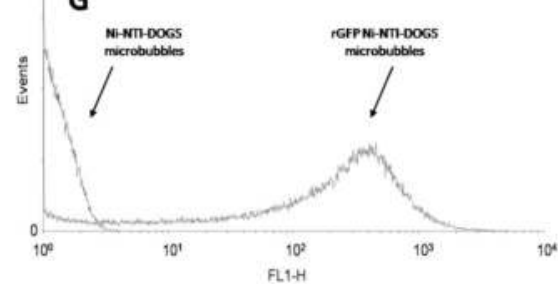

Figure 13. Schematic presentation of metallochelating MBs, confocal and electron microscopy of metallochelating MBs and coupling of proteins with HisTag anchor.

The rGFP-HisTag molecule bound onto the surface of metallochelating liposomes was directly detected by TEM $(\mathbf{A}, \mathbf{B})$. Fluorescence of rGFP was used to prove binding of this protein onto the MB (C). The binding did not affect the native conformation of rGFP as demonstrated by the preserved fluorescence characteristics of the protein. Homogenous population of highly fluorescent rGFP MB was detected by flow cytometry (G). Nonspecific binding of rGFP on both DPPC liposomes as well as MB lacking DOGS-NTA-Ni was not observed by flow cytometry and confocal microscopy. Confocal microscopy confirmed co-localisation of bound rGFP-HisTag (green fluorescence) with the lipids forming MB monolayer. The monolayer was marked with LisR-PE (red fluorescence) (D, E, F). Confocal microscopy revealed that a small fraction of MB had also attached liposomes or residual lipid structures of collapsed MB on their surface (as seen in D, E, F). This is in a good agreement with the electron microscopy data (D) (Adapted from [48]).

Concerning the covalent coupling methods, the techniques are basically the same as the ones developed for the attachment of ligands to liposomes [88]. As the covalent coupling does not require foreign proteins or His-tags (Figure 13), the chances of undesired immune response to the ligand are low [84]. The simple covalent coupling with the formation of peptide bond between the carboxyl-carrying MBs and protein aminogroups is achieved by adding carbo- 
diimide chemistry [89]. First, a carboxyl group is activated with carbodiimide in the presence of $\mathrm{N}$-hydroxysulfosuccinimide, forming active ester [84]. The resulting ester then reacts with the protein aminogroup, forming an amide bond. This method is unfortunately unsuitable for clinical settings as well, as to achieve the desired number of targeting molecules per bubble, large quantities of expensive antibodies must be added to the reaction mixture, not to mention that only very few of them are actually coupled by the peptide bonding [73]. An alternative approach of covalent coupling scheme for antibody attachment to MB surface seems to be thiolmaleimide chemistry, developed for targeted liposome applications [90]. In this case, maleimide on the shell is coupled with a thiol group on the ligand, forming thiol-ether [74]. This technique is convenient with respect to a possible oriented coupling; if a ligand has a single thiol, then a single point attachment to the MB shell should retain affinity of the ligand to this target [84]. Overall, selective oriented coupling with of thiol-protein with a maleimide-carrying MB seems more attractive.

Despite the possibility of attaching the ligand directly to the MB surface, it might seem more suitable to connect the ligand to lipid via an extended spacer arm, such as poly(ethylene glycol) known as PEG. Up to $30 \mathrm{~nm}$ long [91], flexible extended PEG spacer arm separates the ligand and the anchor from each other [92], so that the spacer arm acts as a "fishing line" [93], improving the chances of reaching its target and $\mathrm{MB}$ adhesion and retention on the receptor-coated surfaces $[73,92,94]$. On the other hand, similar to the design of long-circulating liposomes, PEG chains, or PEG chain derivatives, can be incorporated into the shell of MBs in order to form a steric barrier against coalescence and adsorption of macromolecules, such as antibodies [47, 95]. In recent papers, the protective role by the PEG chains was shown when the targeting ligand was attached to shorter PEG chains that were shielded by a PEG overbrush layer (buried-ligand architecture), significantly increasing the MB in vivo stability, prolonging their circulation lifetime and reducing ligand-mediated immunogenicity; it was also reported that the extent of PEG protection to depended on the overbrush length [48, 96-99]. PEG overbrush architecture (PEG - polyethyleneimine with a high degree of substitution) was lately used for complexing and stabilizing the plasmid as well; as a result, the transfection was significantly enhanced [100]. One of the possible reasons for enhanced drug and gene delivery when PEG overbrush strategy is applied is the fact that targeting ligands are generally affixed to the free ends of the PEG chains to direct the MBs to a given target [97], so that the bubbles usually bear thousands of ligands on their surface. Thus cooperative binding of multiple ligands to a target might increase the affinity of particles to the target site, boosting the transfection efficacy. A combination of targeted MB agent BR55 and peptide ligand attached to the bubble shell via a PEG spacer are has even been applied in a prostate cancer clinical trial [101].

\section{Drug- and gene-loading strategies}

The main advantage of the use of MB-based particles as a delivery tool for drugs and genes lies in the dose reduction while maintaining its therapeutic efficacy; the method thus provides much less toxicity than conventional cytotoxic therapies and at the same time has more specificity, which has a great potential particularly in cancer treatment [84]. Generally, drugs 
and/or genes can be either (a) co-administered with MBs, (b) enclosed within the MB shell, (c) incorporated in the MB shell, (d) attached to the surface of the MBs (e) incorporated in liposomes or nanoparticles that are associated with the MB surface.

\subsection{Co-administration of drugs with MBs}

In this case, the MBs enhance the permeability of the endothelial wall in different manners; (1) by rupture of the endothelial wall, so that larger particles such as erythrocytes and polymer particles are able to extravasate [102]; (2) by MBs causing the temporary membrane opening when the low pressure is applied [103]; (3) by sonoporation, allowing for radionuclides or plasmid DNA to pass through [104, 105]. For more detailed description of the mechanisms concerned, please see the above part "Microbubbles in the Ultrasound Field".

\subsection{Enclosing drugs within the MB shell}

Instead of co-injecting drugs and MBs, the bubbles might be modified to contain drugs or DNA $[97,106-110]$. It is virtually possible to load the drugs within the interior of the MBs, which is very convenient since the drug is completely protected and is not released before applying the ultrasound [23]. However, since the MBs are relatively small, the space for drug loading is quite limited. This approach was applied for the first time in 1998 already by the team of Unger, who used lipid-shelled MB with an addition of paclitaxel containing oil phase. Nonetheless, Unger reported the methodology seems to be unsuitable for clinical setting for three reasons; firstly, as the monolayer of the lipid-shelled MB is very thin, only small amount of drug can be loaded; secondly, this strategy is limited only to hydrophobic drugs; last but not least, due to the very high mechanical indexes of ultrasound waves necessary for drug release, which resulted only in $30 \%$ of cell death, the in vivo use of such ultrasound setting appears unfeasible [110].

Recently, Kooiman et al. used oil-filled polymer microcapsule model for ultrasound-mediated delivery of lipophilic drugs; microcapsules with a shell of fluorinated end-capped poly(L-lactic acid) contained hexadecane oil as a drug-carrier reservoir. At higher diagnostic pressures, the microcapsules cracked and released the drug [111]. Despite offering a great potential in clinical use, there are limitations as this approach can be applied only for lipophilic drugs. In another paper, Tinkov et al. suggested the application of doxorubicin (DOX)-loaded phospholipid MBs for targeted tumour therapy. DOX as a potent chemotherapy was loaded into the DPPC/DPPG/ PEG $^{2000}$-DPPE MBs and was tested in a pancreas carcinoma model of the rat. Although an agglomeration and capillary adhesion with embolisation/occlusion of the pulmonary arteries/ capillaries after intravenous infusion of MBs with any new formulation might appear, in this case no rat died or showed any signs of pulmonary vascular occlusion [112]. Therefore the strategy seems suitable for more extensive preclinical therapeutic studies.

\subsection{Nucleic acids and MBs}

Concerning gene delivery, [113] incorporated plasmid DNA inside the gas core of polymer MBs, so that the plasmid was even protected from the host nucleases; at the same time high plasmid load per bubble was achieved [113]. In a recent study, Sun et al. compared the 
transfection efficiency of commercial Definity MBs to modified cationic MBs with longer lipid chain; as a result, cationic bubbles were binding significantly more DNA, so that multi-fold enhancement was reached in gene delivery [114]. The results were consistent with the findings of Panje and Nomikou, et al. and the notion that gene delivery might be augmented with a higher local DNA concentration [115, 116]. In another study, Florinas et al. designed a nonviral siRNA gene carrier using a combination of an arginine-grafted bioreducible polymer (ABP), MBs and ultrasound, for targeting vascular endothelial growth factor (VEGF) in human ovarian cell line; as a result, MBs in combination with lipoplexes showed significantly higher loading capacity compared to naked siRNA; furthermore, only siRNA complexes with ABP and MBs showed significant knock down in human ovarian cell line compared to naked siRNA when incubated for a short time after sonication treatment [116]. The potential of MBs as gene carriers is still on its rise, offering a wide range of options by functionalization of the bubbles.

The drugs that have sufficient affinity for the lipid monolayer might be, in principle, incorporated directly in lipid-shelled MBs [106]. However, due to the fact that the lipid monolayer is rather thin, the amount of drug available for incorporation is very low. Furthermore, the thin shell might not be able to prevent the leakage of drugs from the bubble during their circulation. Therefore other strategies were developed, such as preparation of MBs with a thick lipid shell containing triglyceride oil phase and drug dissolved in it [117]. Nevertheless, this approach is restricted only to hydrophobic fat-soluble drugs, e.g. paclitaxel. A thick polymer shell represents another variety for drug incorporation suitable for water-soluble materials such as DNA and proteins.

Electrostatic interactions of the lipids and plasmid DNA (possessing an overall negative charge) are made possible by the positively charged groups of some synthetic lipids within the shell [109]. DNA can be loaded on the charged MB shell by simply mixing a plasmid with the lipid bubbles before use. After insonation, the surface-coupled DNA remains intact and at the same time is protected against enzymatic degradation $[118,119]$. Moreover, the transfection efficiency is increased by the adherence of DNA to the MBs in comparison with co-administration of MBs and non-adsorbed DNA. At the same time, unbound plasmids should not be able to penetrate into non-targeted cells and tissues and should be degraded, resulting in high specificity with a low transfection in non-insonated regions [19]. Nevertheless, in comparison with the payload of DNA inside the gas core of the bubbles, the loading capacity is restricted to their surface area, which means that high numbers of MBs must be injected. The development of multi-layered assemblies by sequential adsorption of DNA on the positive MB template offers another alternative; because DNA can be directly incorporated as an anionic layer within these assemblies, layer-by-layer methods allow precise control over the loading (or dose) of DNA by controlling film thickness or the number of layers deposited during the fabrication. Besides that, these methods are entirely aqueous and, unlike conventional methods for the encapsulation of DNA in thin polymer films, do not require the use of organic solvents which residua could be presented in these materials post-assembly. [120].

In conclusion, an important advantage of ultrasound-mediated MB-based transfection is the ability to attain DNA delivery and expression only in the insonated areas and not in nontargeted regions. Even if the transfection efficacy is not very high but the encoded protein is 
produced in the target tissue in an amount sufficient to achieve functional biological response, therapeutic intervention should meet its success [121]. Considering the improvement of transfection efficacy via implementation of viral particles using positively charged assemblies, a significant transfection was observed [122]. However, one should be always careful since undesired immune responses after systemic administration might appear.

\subsection{Incorporation of drug loaded LNPs with MBs}

An intensive research has been devoted to the development of nanocarrier-MB hybrids, wherein drug-containing LNPs are physically attached to the surface of MB [21]. These hybrids therefore possess a high drug loading capacity of the nanocarriers and the systemic targeting capability and vascular permeabilizing effect of bubbles. Lum et al. were the first to attach loaded LNPs to MBs via avidin-biotin interaction; they reported on biotinylated lipid MBs to which avidinylated polystyrene beads were attached [117]. The biotinylated MBs were prepared by inclusion of DSPE-PEG-biotin in the lipid shell. Since up to $10^{5} \mathrm{LNPs}$ were bound to each of the bubbles, the drug loading capacity is reasonably enhanced [106]. LNPs seem highly advantageous as (1) they have been long proved as versatile drug delivery systems; (2) a plethora of knowledge is available on LNPs for drug delivery; (3) some LNPs are perfectly and already used in clinical practice. They might also enclose both amphiphilic and hydrophobic drugs in their lipid bilayer, as well as hydrophilic drugs in an aqueous core. Nevertheless, owing to the fact that biotin-avidin linkage might develop an undesired immune response, this approach is not suitable for clinical use. Therefore Geers et al. prepared doxorubicin (DOX)-LNPs loaded MBs containing pDNA or siRNA that in combination with ultrasound boosted both DOX cytotoxicity and pDNA and siRNA delivery to the cells in vitro $[108,119,123,124]$. In their last work, Geers et al. developed a self-assembly of functionalized phospholipids, DOX-loaded LNPs and perfluorobutan gas [125]. Compared to the other methods discussed above to load MBs with drugs, this strategy seems the most advantageous from the loading capacity point of view; though it was estimated that approximately 600 to 1300 LNPs might be bound per singe MB, the amount of loaded DOX is still rather limited. On the other hand, Klibanov et al. prepared liposome-MB pendants incorporating calcein, a hydrophilic self-quenching fluorescent dye, or thrombin, a crucial enzyme of the thrombosis cascade, and they achieved a significant payload volume - up to several cubic micrometres of internal liposome aqueous core space per bubble-LNP [126].

A different approach was introduced by Fan et al. who developed a novel DOX-loaded MB containing super-paramagnetic iron oxide (SPIO) nanoparticles for dual modality imaging and improved image-guided drug delivery [127]. The authors showed that the delivery of SPIO to brain tumours was improved using magnetic targeting in combination with focused ultrasound. Unfortunately, the improvement of DOX delivery using magnetic targeting was not the object of assessment. Hernot et al. prepared nanobody-targeted MBs using metabolic and site-specific biotinylation of nanobodies [128]. The researchers developed a system of carmelidderived single-domain antibody-fragments, i.e. molecular tracers, coupled to targeted MBs. Although they demonstrated the functionality of these modified MBs both in vitro and vivo, since the biotinylated nanobodies were linked to the surface of biotinylated MBs via streptavidin linker, these are not suitable for clinical application due to the undesired immune response. 


\subsection{Infusion of MBs}

Besides imaging applications, MBs showed to be a suitable tool for therapeutic use, e.g. targeted drug delivery, gene therapy, thrombolysis, and vascular permeability enhancement [129]. Certain US-contrast-imaging or therapeutic strategies, such as destruction-reperfusion or MB-enhanced drug delivery, require a continuous injection of the MBs over several minutes. Results of previous in vitro, animal, and human studies have shown that continuous infusion of a US contrast agent can markedly prolong vascular Doppler US enhancement. [130-132]. Most quantitative imaging strategies rely on the ability to administer a consistent dose of contrast agent. The spatial distribution of MBs inside a syringe varies over time owing to the buoyancy of the MBs. These changes are rapid and result in significant inhomogeneity in the concentration and size distribution of the MBs during continuous infusion. The population of MBs that is pumped from a horizontal syringe outlet differs from the initial population as the MBs float to the top of the syringe [133]. This effect is often overlooked and underestimated by experimenters. We believe that this is the main reason for contradictory results published by several authors that compare bolus versus continuous infusion methods of MB application [131]. A superior signal contrast of liver metastasis has been achieved by a continuous application of MBs to patients. US contrast agents for hepatic imaging are traditionally injected as a bolus.

For i.v. applications, precise concentration and size distribution of $\mathrm{MBs}$ is required. Continuous application of MBs might present a problem, owing to their strong tendency to float and accumulate directly below the surface. The concentration and size distribution of specific MBs injected into a patient's blood vessel can be quite different from the ones in the syringe.Recently, we have described the device called Infucon for continuous infusion of MBs which prevents flotation of MBs and their accumulation below the surface. The device has been tested on New Zealand White rabbits using both commercial Sono-Vue diagnostic MBs and PEGylated DPPC MBs prepared in our laboratory $[48,134]$.

The Infucon device consists of several parts: a programmable syringe pump (e.g. LAMBDA VIT-FIT Brno, Czech Republic) with a syringe filled with $\mathrm{SF}_{6}$, an Infucon stirred vessel positioned on magnetic stirrer (e.g. Heidolph MR 3000 D, Germany) and fixed in the position by a holder, and the cannula for application of MBs into the rabbit via ear vein. The schemes of the device together with its photographs are presented in Figure. 14. The role of the syringe pump is to force the gas (e.g. $\mathrm{SF}_{6}$ ) into the Infucon stirred vessel and to inject the MBs into bloodstream via cannula. The same gas which is used for filling MBs is used in the syringe to ensure stability of the MBs during the infusion. The main function of the turbine-like stirrer (which contains two teflon-coated ferrits) is to maintain a uniform dispersion of the MBs in the solution and to prevent their accumulation to the surface owing to their flotation. The stirrer is hung up on the collar of the carrying-off capillary outlet. The direction of rotation is selected to maintain down-stream movement of the fluid and to strip MBs from the surface. The device itself is designed to be hermetically insulated using an O-ring seal. The cell is made of inert materials (Teflon ${ }^{\circledR}$, polypropylene, glass, and Viton ${ }^{\circledR}$ ). All parts of the Infucon are compatible with chemical or heat sterilization. The filter installed in the Infucon head prevents possible air-born particles from entering the cell. The vial (conical bottom) is filled up with a solution 
of MBs (up to $1.5 \mathrm{~mL}$ ) via an inlet capillary. The flow rate (usually in the range of $100-200 \mu \mathrm{L} /$ $\mathrm{min}$ ) and the time of pump action are programmable parameters. The silicon piston of the syringe is properly lubricated to ensure very low friction and smooth movement preventing sudden jumps of the piston resulting in pulsation. A turbine-like stirrer fits the conical bottom of the vial, therefore very low dead volume of residual MB solution is obtained. The turbinelike stirrer freely hangs on the fringe of the Teflon outlet capillary. The capillary is tightly embedded into the Teflon body in order to be prevented from bending. There is also an inlet aperture for incoming gas in this Teflon body. The gas is delivered from the syringe through the inlet capillary linked tightly to the head part of the Infucon (Figure 15, 16). The stirred Infucon cell represents solution of the problem of the continuous infusion of MBs. This device is independent on mechanical stirring of the infusion syringe. Unlike the system based on the rotation of the entire syringe, Infucon cell provides a genuinely homogeneous MB dispersion. The MBs are prevented from flotation by stirring the suspension in a separate cell. The infusion is driven by inert gas filled in a syringe placed in the infusion pump. The inert gas used both for filling the MBs and driving the MB infusion is supposed to have stabilizing effect on the MBs during the process of infusion.
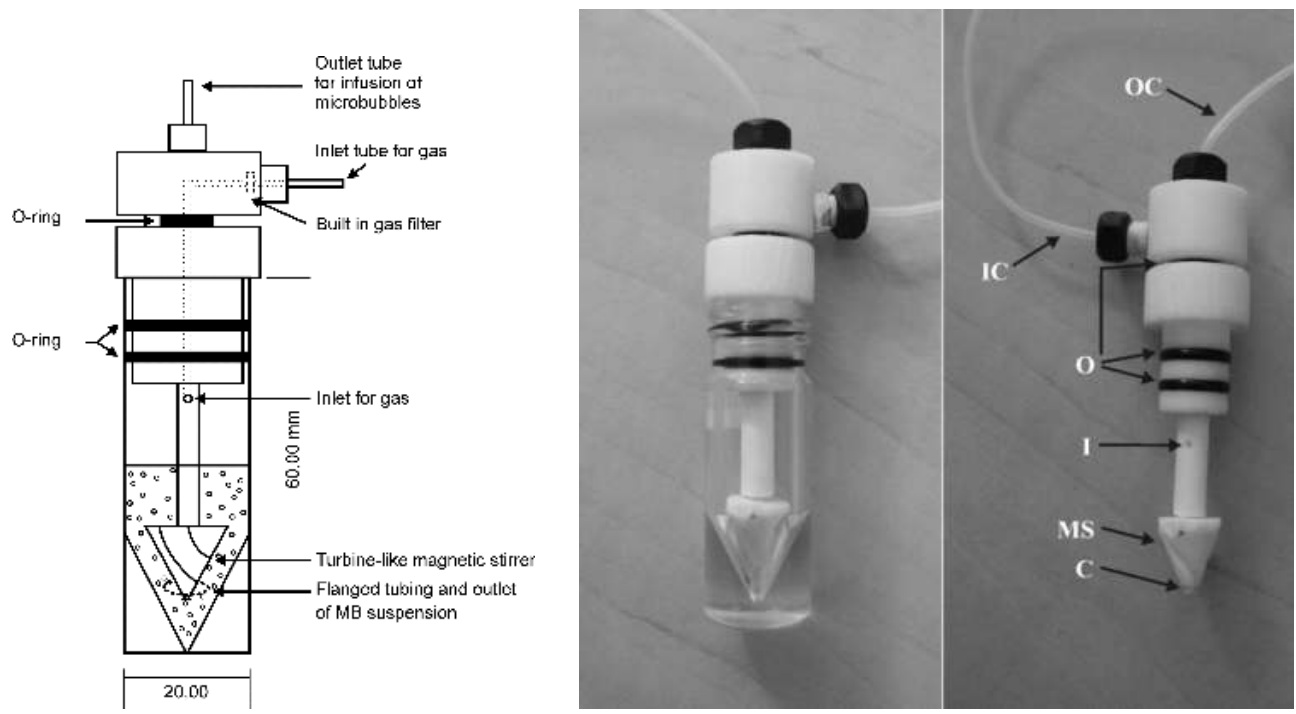

\footnotetext{
(A) Assembled device, (B) Head of the Infucon. (Adapted from [144])

OC - outlet capillary for the infusion of microbubles (connection to the cannula)

IC - inlet capillary for inert gas from a syringe inserted into the syringe pump

$\mathrm{O}$ - O-ring

I - inlet for gas

MS - turbine-like magnetic stirrer with blades

$\mathrm{C}$ - fringe of the outlet capillary (OC)
}

Figure 14. Schematic drawing of the stirred continuous injector Infucon and the photograph of the Infucon device. 

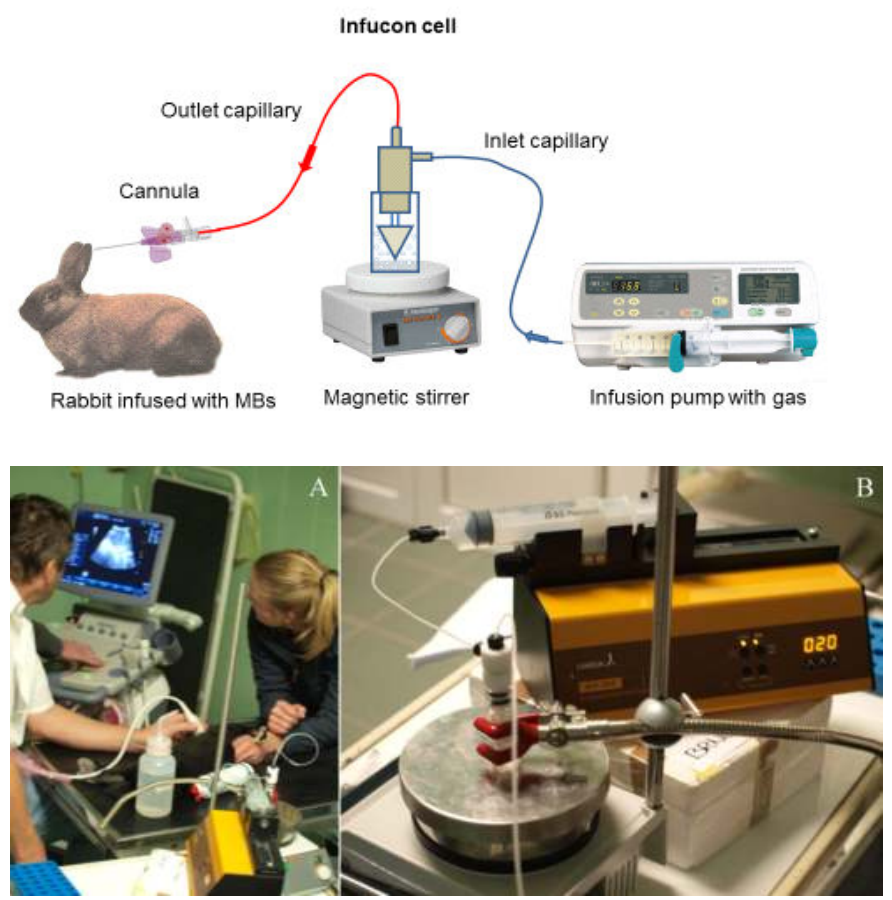

Blue line: gass pushing homogenaous dispersion of MBs

Red line: homogenaous dispesion of MBs driving from Infucon cell into rabbit ear vein via canula

Figure 15. Schematic and real view of the device used for i.v. infusion of MBs into the rabbit.

a. The use of Infucon device during the ultrasound scanning of the rabbit heart.

b. Integration of the Infucon device which is injecting the $\mathrm{MB}$ suspension via vena aricularis into the bloodstream of the rabbit. (Adapted from [144])

\section{Medicinal application}

Imaging the liver - This clinical application of MBs in radiology is most promising. Some portion of MBs is taken up by the liver and spleen. The precise mechanism is hitherto uncertain, but the involvement of the reticulo-endothelial system is probable. In clinical trials, this liver phase generally lasts about 30 minutes when the licensed agent Levovist is used and a few hours with some agents. The liver is very well seen with MB-specific imaging models, such as harmonic imaging, during this phase. The primary practical importance is that numerous focal liver lesions, especially metastases and hepatocellular carcinoma, appear as defects and the use of MBs considerably increases their visibility [135-137]. 
Imaging the heart - MBs can enhance Doppler flow signals in cardiac ultrasonography, and this can be useful in several situations, such as detecting valvular stenoses. The left ventricular cavity is highlighted by MB contrast agents and thus the blood-tissue boundary becomes much clearer. This is useful in detecting abnormalities in wall motion in a given region, assessment of ejection fraction, and finding left ventricular thrombus. The evaluation of left ventricular function is crucial for managing of a number of heart diseases. Both resting and inducible ischaemia can be accurately diagnosed by real time perfusion imaging in stress echocardiography which can be further enhanced by applying intermittent high power pulses destroying the majority of the MBs in a scan plane and subsequently watch refilling: the rate at which it occurs is a measure of microcirculatory flow speed [138].
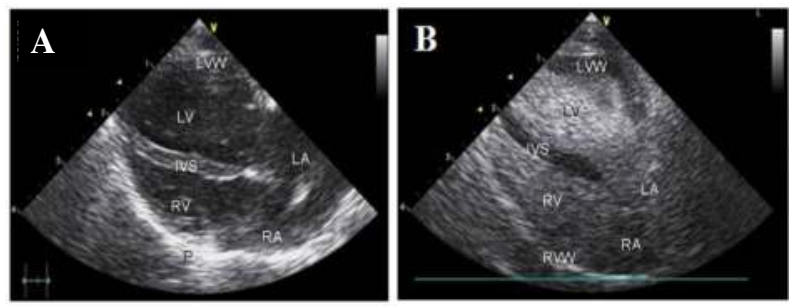

(A) control - an image of the heart without MBs.

(B) Heard filled by MBs during 10 minutes of continual infusion

Figure 16. The sonographs of the rabbit heart before and after the continual infusion of the pegylated micribubbles.Right parasternal long-axis window, 4 - chamber view:LA = left atrium, LV = left ventricle, RA = right atrium, $R V$ = right ventricle, IVS = intervetricular septum, LVW = left ventricular wall, $R V W=$ right ventricular wall, $\mathrm{P}=$ pericardium - lung interface. (Adapted from [144])

\section{Therapeutic applications recent advances in molecular, multimodal and theranostic ultrasound imaging}

The importance of MBs usage in treatment is higher than their use as a diagnostic tool. At present, the different characteristic features of MBs (various sizes and the types of gases and shell materials) precondition them for use as targeting drug carriers. MBs can help in drug delivery in themselves (by acting as "cavitation nuclei") and as drug carriers for site-specific treatment. Due to these characteristics they can be employed as drug carriers for treatment of various diseases, such as inflammation, thrombus, lymph nodes, cancer, angiogenesis and atherosclerosis [136].

\subsection{Gene therapy}

The use of MBs in gene therapy is most challenging. The delivery of genetic material to a selected site represents the main problem in this emerging area. Drug delivery can be potentiated by ultrasound which creates transient non-lethal perforations in cell membranes facilitating penetration of large molecules and particles into the cells ("sonoporating"). During this process, high acoustic power is necessary, which is beyond that permitting for imaging. 
However, in the presence of MBs the amount of required power is substantially reduced because the MBs decrease the amount of energy needed for the process of cavitation, leading to $\mathrm{MB}$ collapse due to excessive oscillations induced by ultrasound pulses. Furthermore, cavitation of MBs in capillary beds leads to a capillary permeability increase, thus improving local access of the released therapeutic drug [139]. The clinical use of virus-based vectors for gene therapy is limited. This is due to the fact that immune response can be induced by viral proteins in the target tissue and elicit a strong inflammatory activation of endothelial cells. However, it has been shown that when e.g. plasmids and antisense oligonucleotides are used in non-viral delivery vehicles, transfection efficiency is decreased and the expression of the gene product is transient. Commonly used MB preparation Levovist enhanced the beta-Gal gene transfection efficiency more than 6-fold and Optison were used for gene transfer to cardiomyocytes. The transfection efficiencies are significantly higher than that of no $\mathrm{MB}$ and it is similar to Levovist $[97,100,114]$.

\subsection{Blood-brain barrier}

The blood-brain barrier (BBB) is a specialized system of capillary endothelial cells preventing harmful substances in the blood stream from entering the brain. They also supply the brain with nutrients necessary for proper brain functioning. The BBB regulates transport through both tight junctions and metabolic barriers and is often a rate-limiting factor in determining permeation of therapeutic drugs into the brain. It is a significant obstacle affecting delivery of both small molecules and macromolecular agents. Despite the fact that a variety of drugs could be potentially used to treat brain diseases, no method exists that would allow non-invasivetargeted delivery through the BBB.

Ultrasound can be used to locally deliver a drug or gene to a specific region of interest in the brain. The benefit of the MB technique is that it can be used on a very small area of the BBB. MBs are used in combination with ultrasound exposure. The effects of ultrasound can be focused upon the vasculature to reduce the acoustic intensity required for BBB opening production. Several pathways of transcapillary passage after ultrasound sonication have been recognized, including transcytosis, passage through endothelial cell cytoplasmic openings holeyed by "nanoschrapnels" produced by explosion of $\mathrm{MB}$, opening of tight junctions by oscillating MBs and free passage through injured endothelium [136, 140, 141].

\subsection{Thrombolysis and thrombus destruction}

The major cause of death in Western countries is thrombo-occlusive cardiovascular disease, with acute ST-elevation myocardial infarction (STEMI) being a serious medical condition leading to high morbidity and mortality. Two main treatment strategies have been developed during the past years. Their aim is to immediately restore coronary blood flow. In the first studies it was shown that fibrinolysis may be accelerated by ultrasound alone. Besides that, fibrinolysis can be even more increased by the addition of MBs which are destroyed by ultrasound induced high pressure. Three mechanisms leading to this increase have been proposed. The first is the occurrence of complete stress based on micro-jets formed after the destruction of the MBs by ultrasound. The second mechanism is the generation of reactive oxygen species in endothelial cells. The third proposed mechanism is a temperature increase, 
also occurring after the MBs collapse induced by ultrasound exposure. Whereas the full mechanism still remains to be elucidated, evidence indicates that the use of ultrasound alone and ultrasound combined with MBs can enhance clot dissolution by creating microholes in freshly formed thrombi $[142,143]$.

\section{Conclusions}

LNPs and MBs are examples of the successful transfer of basic and applied research into commercial medicinal products. Biocompatibility and biodegradability of LNPs together with versatility in modification and functionalization are features which put together favour their application in medicine. Combinations of LNPs and MBs make best use of both technologies to form new theranostic systems that could form the basis of future advanced therapeutics.

\section{Acknowledgements}

The chapter was prepared within collaborative projects: Centre of Excellence for International Research CZ.1.07/2.3.00/20.0164 to JT, MR and ADM. We gratefully acknowledge the support by Project Centre of Excellence for Nanotoxicology GACR GAP503/12/G147. Štěpán Koudelka was supported by European Regional Development Fund - Project FNUSA-ICRC (No. CZ. 1.05/1.1.00/02.0123), European Social Fund and the State Budget of the Czech Republic - Project FNUSA - ICRC - Support for neurological R\&D teams through new postdoc positions formation (No. CZ.1.07/2.3.00/30.0043). The author's team was supported by the project FIT.

The support by Global Acorn is kindly acknowledged and appreciated.

\section{Author details}

Jaroslav Turánek ${ }^{1 *}$, Andrew D. Miller ${ }^{2,3}$, Zuzana Kauerová ${ }^{1}$, Róbert Lukáč ${ }^{1}$, Josef Mašek ${ }^{1}$, Štěpán Koudelka ${ }^{1,4}$ and Milan Raška ${ }^{5}$

*Address all correspondence to: turanek@seznam.cz

1 Veterinary Research Institute, Brno, Czech Republic

2 Institute of Pharmaceutical Science, King's College London, London, UK

3 GlobalAcorn Ltd, London, UK

4 International Clinical Research Center, St. Anne's University Hospital, Brno, Czech Republic

5 Department of Immunology, Faculty of Medicine and Dentistry, Palacky University, Olomouc, Czech Republic 


\section{References}

[1] Miller A, Tanner J. Essentials of chemical biology : structure and dynamics of biological macromolecules. Chichester: John Wiley \& Sons; 2008.

[2] Miller AD. Delivery of RNAi therapeutics: work in progress. Expert review of medical devices. 2013;10(6) 781-811.

[3] Miller AD. Delivering the promise of small ncRNA therapeutics. Therapeutic delivery. 2014;5(5) 569-89.

[4] Escriou V, Mignet N, Miller AD. Auto-associative lipid-based systems for nonviral nucleic acid delivery. In: Scherman D, editor. Advanced Textbook on Gene Transfer, Gene Therapy and Genetic Pharmacology. London: Imperial College Press; 2014. p. 221-54.

[5] Miller AD. Lipid-based nanoparticles in cancer diagnosis and therapy. Journal of drug delivery. 2013; 165981.

[6] Davis SS. Coming of age of lipid-based drug delivery systems - Commentary. Advanced drug delivery reviews. 2004;56(9) 1241-2.

[7] Lian T, Ho RJY. Trends and developments in liposome drug delivery systems. J Pharm Sci. 2001;90(6) 667-80.

[8] de Menezes DEL, Pilarski LM, Belch AR, Allen TM. Selective targeting of immunoliposomal doxorubicin against human multiple myeloma in vitro and ex vivo. Bba-Biomembranes. 2000;1466(1-2) 205-20.

[9] Modo MMJJ, Bulte JWM. Molecular and cellular MR imaging. Boca Raton: CRC Press; 2007. 421 p. p.

[10] Modo M, Hoehn M, Bulte JW. Cellular MR imaging. Molecular imaging. 2005;4(3) 143-64.

[11] Caravan P, Ellison JJ, McMurry TJ, Lauffer RB. Gadolinium(III) chelates as MRI contrast agents: Structure, dynamics, and applications. Chem Rev. 1999;99(9) 2293-352.

[12] Maeda H, Greish K, Fang J. The EPR effect and polymeric drugs: A paradigm shift for cancer chemotherapy in the 21st century. Adv Polym Sci. 2006;193 103-21.

[13] Iyer AK, Khaled G, Fang J, Maeda H. Exploiting the enhanced permeability and retention effect for tumor targeting. Drug discovery today. 2006;11(17-18) 812-8.

[14] Kamaly N, Miller AD. Paramagnetic liposome nanoparticles for cellular and tumour imaging. Int J Mol Sci, 2010; 11(4), 1759-1776.

[15] Frangioni JV, Hajjar RJ. In vivo tracking of stem cells for clinical trials in cardiovascular disease. Circulation. 2004;110(21) 3378-83. 
[16] Rogers WJ, Meyer CH, Kramer CM. Technology insight: in vivo cell tracking by use of MRI. Nat Clin Pract Card. 2006;3(10) 554-62.

[17] Mukherjee S, Ghosh RN, Maxfield FR. Endocytosis. Physiological reviews. 1997;77(3) 759-803.

[18] Kamaly N, Kalber T, Thanou M, Bell JD, Miller AD. Folate receptor targeted bimodal liposomes for tumor magnetic resonance imaging. Bioconjugate chemistry. 2009;20(4) 648-55.

[19] Hernot S, Klibanov AL. Microbubbles in ultrasound-triggered drug and gene delivery. Advanced drug delivery reviews. 2008;60(10) 1153-66.

[20] Sirsi S, Borden M. Microbubble Compositions, Properties and Biomedical Applications. Bubble science engineering and technology. 2009;1(1-2) 3-17.

[21] Sirsi SR, Borden MA. State-of-the-art materials for ultrasound-triggered drug delivery. Advanced drug delivery reviews. 2014;72 3-14.

[22] Chen JL, Dhanaliwala AH, Dixon AJ, Klibanov AL, Hossack JA. Synthesis and characterization of transiently stable albumin-coated microbubbles via a flow-focusing microfluidic device. Ultrasound in medicine \& biology. 2014;40(2) 400-9.

[23] Lentacker I, De Smedt SC, Sanders NN. Drug loaded microbubble design for ultrasound triggered delivery. Soft Matter. 2009;5(11) 2161-70.

[24] Perkins AC, Frier M, Hindle AJ, Blackshaw PE, Bailey SE, Hebden JM, et al. Human biodistribution of an ultrasound contrast agent (Quantison(TM)) by radiolabelling and gamma scintigraphy. Brit J Radiol. 1997;70(834) 603-11.

[25] Kauerova Z. Targeting nanoparticle drug carriers: Functionalized microbubbles and their application in ultrasonography: Thesis, Masaryk University, Brno; 2013.

[26] Kaya M, Feingold S, Hettiarachchi K, Lee AP, Dayton PA. Acoustic responses of monodisperse lipid-encapsulated microbubble contrast agents produced by flow focusing. Bubble science engineering and technology. 2010;2(2) 33-40.

[27] Streeter JE, Gessner R, Miles I, Dayton PA. Improving sensitivity in ultrasound molecular imaging by tailoring contrast agent size distribution: in vivo studies. Molecular imaging. 2010;9(2) 87-95.

[28] Gorce JM, Arditi M, Schneider M. Influence of bubble size distribution on the echogenicity of ultrasound contrast agents: a study of SonoVue. Investigative radiology. 2000;35(11) 661-71.

[29] Soetanto K, Chan M. Fundamental studies on contrast images from different-sized microbubbles: analytical and experimental studies. Ultrasound in medicine \& biology. 2000;26(1) 81-91.

[30] Klibanov AL. Ultrasound contrast agents: Development of the field and current status. Top Curr Chem. 2002;222 73-106. 
[31] Konofagou EE, Tung YS, Choi J, Deffieux T, Baseri B, Vlachos F. Ultrasound-induced blood-brain barrier opening. Current pharmaceutical biotechnology. 2012;13(7) 1332-45.

[32] Burke CW, Suk JS, Kim AJ, Hsiang YH, Klibanov AL, Hanes J, et al. Markedly enhanced skeletal muscle transfection achieved by the ultrasound-targeted delivery of non-viral gene nanocarriers with microbubbles. J Control Release. 2012;162(2) 414-21.

[33] Andre MP, Steinbach G, Mattrey RF. Enhancement of the echogenicity of flowing blood by the contrast agent perflubron. Investigative radiology. 1993;28(6) 502-6.

[34] Tartis MS, Kruse DE, Zheng H, Zhang H, Kheirolomoom A, Marik J, et al. Dynamic microPET imaging of ultrasound contrast agents and lipid delivery. J Control Release. 2008;131(3) 160-6.

[35] Kabalnov A, Klein D, Pelura T, Schutt E, Weers J. Dissolution of multicomponent microbubbles in the bloodstream: 1. Theory. Ultrasound in medicine \& biology. 1998;24(5) 739-49.

[36] Lentacker I, De Cock I, Deckers R, De Smedt SC, Moonen CT. Understanding ultrasound induced sonoporation: definitions and underlying mechanisms. Advanced drug delivery reviews. 2014;72 49-64.

[37] Lee JL, Lo CW, Ka SM, Chen A, Chen WS. Prolonging the expression duration of ultrasound-mediated gene transfection using PEI nanoparticles. J Control Release. 2012;160(1) 64-71.

[38] Miller DL, Nyborg WL. Theoretical investigation of the response of gas-filled micropores and cavitation nuclei to ultrasound J Acoust Soc Am 1983;73(5) 1537-44.

[39] Davies PF. Flow-mediated endothelial mechanotransduction. Physiological reviews. 1995;75(3) 519-60.

[40] Vokurka K. Comparison of Rayleigh's, Herring's, and Gilmore's models of gas bubbles. Acta Acustica united with Acustica. 1986;59(3) 214-9.

[41] Miller DL, Averkiou MA, Brayman AA, Everbach EC, Holland CK, Wible JH, Jr., et al. Bioeffects considerations for diagnostic ultrasound contrast agents. Journal of ultrasound in medicine : official journal of the American Institute of Ultrasound in Medicine. 2008;27(4) 611-32; quiz 33-6.

[42] Pitt WG, Husseini GA, Staples BJ. Ultrasonic drug delivery--a general review. Expert opinion on drug delivery. 2004;1(1) 37-56.

[43] Sirsi SR, Borden MA. Advances in Ultrasound Mediated Gene Therapy Using Microbubble Contrast Agents. Theranostics. 2012;2(12) 1208-22.

[44] $\mathrm{Wu} \mathrm{J}$, Nyborg WL. Ultrasound, cavitation bubbles and their interaction with cells. Advanced drug delivery reviews. 2008;60(10) 1103-16. 
[45] Mehier-Humbert S, Bettinger T, Yan F, Guy RH. Plasma membrane poration induced by ultrasound exposure: implication for drug delivery. J Control Release. 2005;104(1) 213-22.

[46] van Wamel A, Bouakaz A, Versluis M, de Jong N. Micromanipulation of endothelial cells: ultrasound-microbubble-cell interaction. Ultrasound in medicine \& biology. 2004;30(9) 1255-8.

[47] Klibanov AL. Targeted delivery of gas-filled microspheres, contrast agents for ultrasound imaging. Advanced drug delivery reviews. 1999;37(1-3) 139-57.

[48] Lukac R, Kauerova Z, Masek J, Bartheldyova E, Kulich P, Koudelka S, et al. Preparation of Metallochelating Microbubbles and Study on Their Site-Specific Interaction with rGFP-HisTag as a Model Protein. Langmuir : the ACS journal of surfaces and colloids. 2011;27(8) 4829-37.

[49] Meijering BDM, Juffermans LJM, van Wamel A, Henning RH, Zuhorn IS, Emmer M, et al. Ultrasound and Microbubble-Targeted Delivery of Macromolecules Is Regulated by Induction of Endocytosis and Pore Formation. Circ Res. 2009;104(5) 679-U226.

[50] van Wamel A, Kooiman K, Harteveld M, Emmer M, ten Cate FJ, Versluis M, et al. Vibrating microbubbles poking individual cells: Drug transfer into cells via sonoporation. J Control Release. 2006;112(2) 149-55.

[51] Zhou Y, Yang K, Cui J, Ye JY, Deng CX. Controlled permeation of cell membrane by single bubble acoustic cavitation. J Control Release. 2012;157(1) 103-11.

[52] Ward M, Wu J, Chiu JF. Experimental study of the effects of Optison concentration on sonoporation in vitro. Ultrasound in medicine \& biology. 2000;26(7) 1169-75.

[53] Miller DL. Overview of experimental studies of biological effects of medical ultrasound caused by gas body activation and inertial cavitation. Progress in biophysics and molecular biology. 2007;93(1-3) 314-30.

[54] Juffermans LJ, van Dijk A, Jongenelen CA, Drukarch B, Reijerkerk A, de Vries HE, et al. Ultrasound and microbubble-induced intra- and intercellular bioeffects in primary endothelial cells. Ultrasound in medicine \& biology. 2009;35(11) 1917-27.

[55] Juffermans LJ, Meijering DB, van Wamel A, Henning RH, Kooiman K, Emmer M, et al. Ultrasound and microbubble-targeted delivery of therapeutic compounds: ICIN Report Project 49: Drug and gene delivery through ultrasound and microbubbles. Netherlands heart journal : monthly journal of the Netherlands Society of Cardiology and the Netherlands Heart Foundation. 2009;17(2) 82-6.

[56] Juffermans LJM, Dijkmans PA, Musters RJP, Visser CA, Kamp O. Transient permeabilization of cell membranes by ultrasound-exposed microbubbles is related to formation of hydrogen peroxide. Am J Physiol-Heart C. 2006;291(4) H1595-H601.

[57] Postema M, Schmitz G. Bubble dynamics involved in ultrasonic imaging. Expert review of molecular diagnostics. 2006;6(3) 493-502. 
[58] Dayton PA, Allen JS, Ferrara KW. The magnitude of radiation force on ultrasound contrast agents. The Journal of the Acoustical Society of America. 2002;112(5 Pt 1) 2183-92.

[59] Rychak JJ, Klibanov AL, Hossack JA. Acoustic radiation force enhances targeted delivery of ultrasound contrast microbubbles: in vitro verification. IEEE transactions on ultrasonics, ferroelectrics, and frequency control. 2005;52(3) 421-33.

[60] Fan Z, Sun Y, Di C, Tay D, Chen W, Deng CX, et al. Acoustic tweezing cytometry for live-cell subcellular modulation of intracellular cytoskeleton contractility. Scientific reports. 2013;3 2176.

[61] Ferrara KW. Driving delivery vehicles with ultrasound. Advanced drug delivery reviews. 2008;60(10) 1097-102.

[62] Delalande A, Kotopoulis S, Rovers T, Pichon C, Postema M. Sonoporation at a low mechanical index. Bubble Sci Eng Technol. 2011;3 3-12.

[63] Caskey CF, Qin S, Dayton PA, Ferrara KW. Microbubble tunneling in gel phantoms. The Journal of the Acoustical Society of America. 2009;125(5) EL183-9.

[64] Arvanitis CD, Bazan-Peregrino M, Rifai B, Seymour LW, Coussios CC. Cavitation-enhanced extravasation for drug delivery. Ultrasound in medicine \& biology. 2011;37(11) 1838-52.

[65] Gramiak R, Shah PM. Echocardiography of the aortic root. Investigative radiology. 1968;3(5) 356-66.

[66] Panje CM, Wang DS, Pysz MA, Paulmurugan R, Ren Y, Tranquart F, et al. Ultrasound-mediated gene delivery with cationic versus neutral microbubbles: effect of DNA and microbubble dose on in vivo transfection efficiency. Theranostics. 2012;2(11) 1078-91.

[67] Tachibana K, Tachibana S. The use of ultrasound for drug delivery. Echocardiogr-J Card. 2001;18(4) 323-8.

[68] Frinking PJA, Bouakaz A, Kirkhorn J, Ten Cate FJ, de Jong N. Ultrasound contrast imaging: Current and new potential methods. Ultrasound in Medicine and Biology. 2000;26(6) 965-75.

[69] Ferrara K, Pollard R, Borden M. Ultrasound microbubble contrast agents: fundamentals and application to gene and drug delivery. Annual review of biomedical engineering. 2007;9 415-47.

[70] Wang S, Samiotaki G, Olumolade O, Feshitan JA, Konofagou EE. Microbubble type and distribution dependence of focused ultrasound-induced blood-brain barrier opening. Ultrasound in medicine \& biology. 2014;40(1) 130-7.

[71] Yao L, Song Q, Bai W, Zhang J, Miao D, Jiang M, et al. Facilitated brain delivery of poly (ethylene glycol)-poly (lactic acid) nanoparticles by microbubble-enhanced unfocused ultrasound. Biomaterials. 2014;35(10) 3384-95. 
[72] Tung YS, Vlachos F, Feshitan JA, Borden MA, Konofagou EE. The mechanism of interaction between focused ultrasound and microbubbles in blood-brain barrier opening in mice. Journal of the Acoustical Society of America. 2011;130(5) 3059-67.

[73] Klibanov AL. Preparation of targeted microbubbles: ultrasound contrast agents for molecular imaging. Medical \& biological engineering \& computing. 2009;47(8) 875-82.

[74] Klibanov AL. Molecular imaging with targeted ultrasound contrast microbubbles. Ernst Schering Research Foundation workshop. 2005(49) 171-91.

[75] Klibanov AL, Maruyama K, Beckerleg AM, Torchilin VP, Huang L. Activity of amphipathic poly(ethylene glycol) 5000 to prolong the circulation time of liposomes depends on the liposome size and is unfavorable for immunoliposome binding to target. Biochimica et biophysica acta. 1991;1062(2) 142-8.

[76] Allen TM, Williamson P, Schlegel RA. Phosphatidylserine as a Determinant of Reticuloendothelial Recognition of Liposome Models of the Erythrocyte Surface. P Natl Acad Sci USA. 1988;85(21) 8067-71.

[77] Lindner JR, Song J, Xu F, Klibanov AL, Singbartl K, Ley K, et al. Noninvasive ultrasound imaging of inflammation using microbubbles targeted to activated leukocytes. Circulation. 2000;102(22) 2745-50.

[78] Nakano H, Ishida Y, Hatakeyama T, Sakuraba K, Hayashi M, Sakurai O, et al. Contrast-enhanced intraoperative ultrasonography equipped with late Kupffer-phase image obtained by sonazoid in patients with colorectal liver metastases. World J Gastroentero. 2008;14(20) 3207-11.

[79] Yanagisawa K, Moriyasu F, Miyahara T, Yuki M, Iijima H. Phagocytosis of ultrasound contrast agent microbubbles by Kupffer cells. Ultrasound in Medicine and Biology. 2007;33(2) 318-25.

[80] Korpanty G, Grayburn PA, Shohet RV, Brekken RA. Targeting vascular endothelium with avidin microbubbles. Ultrasound in Medicine and Biology. 2005;31(9) 1279-83.

[81] Korpanty G, Carbon JG, Grayburn PA, Fleming JB, Brekken RA. Monitoring response to anticancer therapy by targeting microbubbles to tumor vasculature. Clinical cancer research : an official journal of the American Association for Cancer Research. 2007;13(1) 323-30.

[82] Bayer EA, Wilchek M, Skutelsky E. Affinity cytochemistry: the localization of lectin and antibody receptors on erythrocytes via the avidin-biotin complex. FEBS letters. $1976 ; 68(2)$ 240-4.

[83] Klibanov AL, Hughes MS, Marsh JN, Hall CS, Miller JG, Wible JH, et al. Targeting of ultrasound contrast material. An in vitro feasibility study. Acta radiologica Supplementum. 1997;412 113-20. 
[84] Unnikrishnan S, Klibanov AL. Microbubbles as ultrasound contrast agents for molecular imaging: preparation and application. AJR American journal of roentgenology. 2012;199(2) 292-9.

[85] Klibanov AL. Ligand-carrying gas-filled microbubbles: ultrasound contrast agents for targeted molecular imaging. Bioconjugate chemistry. 2005;16(1) 9-17.

[86] Krupka M, Masek J, Bartheldyova E, Turanek Knotigova P, Plockova J, Korvasova Z, et al. Enhancement of immune response towards non-lipidized Borrelia burgdorferi recombinant OspC antigen by binding onto the surface of metallochelating nanoliposomes with entrapped lipophilic derivatives of norAbuMDP. J Control Release. 2012;160(2) 374-81.

[87] Watson DS, Endsley AN, Huang L. Design considerations for liposomal vaccines: Influence of formulation parameters on antibody and cell-mediated immune responses to liposome associated antigens. Vaccine. 2012;30(13) 2256-72.

[88] Takalkar AM, Klibanov AL, Rychak JJ, Lindner JR, Ley K. Binding and detachment dynamics of microbubbles targeted to P-selectin under controlled shear flow. J Control Release. 2004;96(3) 473-82.

[89] Villanueva FS, Jankowski RJ, Klibanov S, Pina ML, Alber SM, Watkins SC, et al. Microbubbles targeted to intercellular adhesion molecule- 1 bind to activated coronary artery endothelial cells. Circulation. 1998;98(1) 1-5.

[90] Kirpotin D, Park JW, Hong K, Zalipsky S, Li WL, Carter P, et al. Sterically stabilized anti-HER2 immunoliposomes: design and targeting to human breast cancer cells in vitro. Biochemistry. 1997;36(1) 66-75.

[91] Jeppesen C, Wong JY, Kuhl TL, Israelachvili JN, Mullah N, Zalipsky S, et al. Impact of polymer tether length on multiple ligand-receptor bond formation. Science. 2001;293(5529) 465-8.

[92] Kim DH, Klibanov AL, Needham D. The influence of tiered layers of surface-grafted poly(ethylene glycol) on receptor-ligand-mediated adhesion between phospholipid monolayer-stabilized microbubbles and coated class beads. Langmuir : the ACS journal of surfaces and colloids. 2000;16(6) 2808-17.

[93] Lee RJ, Low PS. Folate-Mediated Tumor-Cell Targeting of Liposome-Entrapped Doxorubicin in-Vitro. Bba-Biomembranes. 1995;1233(2) 134-44.

[94] Ham AS, Klibanov AL, Lawrence MB. Action at a distance: lengthening adhesion bonds with poly(ethylene glycol) spacers enhances mechanically stressed affinity for improved vascular targeting of microparticles. Langmuir : the ACS journal of surfaces and colloids. 2009;25(17) 10038-44.

[95] Klibanov AL, Maruyama K, Torchilin VP, Huang L. Amphipathic polyethyleneglycols effectively prolong the circulation time of liposomes. FEBS letters. 1990;268(1) 235-7. 
[96] Chen CC, Borden MA. The role of poly(ethylene glycol) brush architecture in complement activation on targeted microbubble surfaces. Biomaterials. 2011;32(27) 6579-87.

[97] Unger E, Porter T, Lindner J, Grayburn P. Cardiovascular drug delivery with ultrasound and microbubbles. Advanced drug delivery reviews. 2014;72 110-26.

[98] Moghimi SM, Szebeni J. Stealth liposomes and long circulating nanoparticles: critical issues in pharmacokinetics, opsonization and protein-binding properties. Progress in lipid research. 2003;42(6) 463-78.

[99] Cavalli R, Bisazza A, Lembo D. Micro- and nanobubbles: a versatile non-viral platform for gene delivery. International journal of pharmaceutics. 2013;456(2) 437-45.

[100] Rychak JJ, Klibanov AL. Nucleic acid delivery with microbubbles and ultrasound. Advanced drug delivery reviews. 2014;72 82-93.

[101] Pillai R, Marinelli ER, Fan H, Nanjappan P, Song B, von Wronski MA, et al. A phospholipid-PEG2000 conjugate of a vascular endothelial growth factor receptor 2 (VEGFR2)-targeting heterodimer peptide for contrast-enhanced ultrasound imaging of angiogenesis. Bioconjugate chemistry. 2010;21(3) 556-62.

[102] Price RJ, Skyba DM, Kaul S, Skalak TC. Delivery of colloidal, particles and red blood cells to tissue through microvessel ruptures created by targeted microbubble destruction with ultrasound. Circulation. 1998;98(13) 1264-7.

[103] Marmottant P, Hilgenfeldt S. A bubble-driven microfluidic transport element for bioengineering. P Natl Acad Sci USA. 2004;101(26) 9523-7.

[104] Mayer CR, Bekeredjian R. Ultrasonic gene and drug delivery to the cardiovascular system. Advanced drug delivery reviews. 2008;60(10) 1177-92.

[105] van Wamel A, Bouakaz A, Bernard B, ten Cate F, de Jong N. Radionuclide tumour therapy with ultrasound contrast microbubbles. Ultrasonics. 2004;42(1-9) 903-6.

[106] Bohmer MR, Klibanov AL, Tiemann K, Hall CS, Gruell H, Steinbach OC. Ultrasound triggered image-guided drug delivery. European journal of radiology. 2009;70(2) 242-53.

[107] Shi WT, Bohmer M, van Wamel A, Celebi M, Klibanov AL, Chin CT, et al. Ultrasound therapy with drug loaded microcapsules. Ultrason. 2007 773-6.

[108] Vandenbroucke RE, Lentacker I, Demeester J, De Smedt SC, Sanders NN. Ultrasound assisted siRNA delivery using PEG-siPlex loaded microbubbles. J Control Release. 2008;126(3) 265-73.

[109] Lentacker I, De Smedt SC, Demeester J, Sanders NN. Microbubbles which bind and protect DNA against nucleases. J Control Release. 2006;116(2) E73-E5. 
[110] Unger EC, McCreery TP, Sweitzer RH, Caldwell VE, Wu Y. Acoustically active lipospheres containing paclitaxel: a new therapeutic ultrasound contrast agent. Investigative radiology. 1998;33(12) 886-92.

[111] Kooiman K, Bohmer MR, Emmer M, Vos HJ, Chlon C, Shi WT, et al. Oil-filled polymer microcapsules for ultrasound-mediated delivery of lipophilic drugs. J Control Release. 2009;133(2) 109-18.

[112] Tinkov S, Coester C, Serba S, Geis NA, Katus HA, Winter G, et al. New doxorubicinloaded phospholipid microbubbles or targeted tumor therapy: in-vivo characterization. J Control Release. 2010;148(3) 368-72.

[113] Hauff P, Seemann S, Reszka R, Schultze-Mosgau M, Reinhardt M, Buzasi T, et al. Evaluation of gas-filled microparticles and sonoporation as gene delivery system: Feasibility study in rodent tumor models. Radiology. 2005;236(2) 572-8.

[114] Sun RR, Noble ML, Sun SS, Song S, Miao CH. Development of therapeutic microbubbles for enhancing ultrasound-mediated gene delivery. J Control Release. 2014;182 111-20.

[115] Nomikou N, Tiwari P, Trehan T, Gulati K, McHale AP. Studies on neutral, cationic and biotinylated cationic microbubbles in enhancing ultrasound-mediated gene delivery in vitro and in vivo. Acta biomaterialia. 2012;8(3) 1273-80.

[116] Florinas S, Nam HY, Kim SW. Enhanced siRNA Delivery Using a Combination of an Arginine-Grafted Bioreducible Polymer, Ultrasound, and Microbubbles in Cancer Cells. Mol Pharmaceut. 2013;10(5) 2021-30.

[117] Lum AFH, Borden MA, Dayton PA, Kruse DE, Simon SI, Ferrara KW. Ultrasound radiation force enables targeted deposition of model drug carriers loaded on microbubbles. J Control Release. 2006;111(1-2) 128-34.

[118] Christiansen JP, French BA, Klibanov AL, Kaul S, Lindner JR. Targeted tissue transfection with ultrasound destruction of plasmid-bearing cationic microbubbles. Ultrasound in medicine \& biology. 2003;29(12) 1759-67.

[119] Lentacker I, De Geest BG, Vandenbroucke RE, Peeters L, Demeester J, De Smedt SC, et al. Ultrasound-responsive polymer-coated microbubbles that bind and protect DNA. Langmuir : the ACS journal of surfaces and colloids. 2006;22(17) 7273-8.

[120] Jewell CM, Lynn DM. Multilayered polyelectrolyte assemblies as platforms for the delivery of DNA and other nucleic acid-based therapeutics. Advanced drug delivery reviews. 2008;60(9) 979-99.

[121] Leong-Poi H, Kuliszewski MA, Lekas M, Sibbald M, Teichert-Kuliszewska K, Klibanov AL, et al. Therapeutic arteriogenesis by ultrasound-mediated VEGF165 plasmid gene delivery to chronically ischemic skeletal muscle. Circ Res. 2007;101(3) 295-303. 
[122] Taylor SL, Rahim AA, Bush NL, Barnber JC, Porter CD. Targeted retroviral gene delivery using ultrasound. J Gene Med. 2007;9(2) 77-87.

[123] Lentacker I, De Smedt SC, Demeester J, Van Marck V, Bracke M, Sanders NN. Lipoplex-loaded microbubbles for gene delivery: A Trojan horse controlled by ultrasound. Adv Funct Mater. 2007;17(12) 1910-6.

[124] Lentacker I, Vandenbroucke RE, Lucas B, Demeester J, De Smedt SC, Sanders NN. New strategies for nucleic acid delivery to conquer cellular and nuclear membranes. J Control Release. 2008;132(3) 279-88.

[125] Geers B, Lentacker I, Sanders NN, Demeester J, Meairs S, De Smedt SC. Self-assembled liposome-loaded microbubbles: The missing link for safe and efficient ultrasound triggered drug-delivery. J Control Release. 2011;152(2) 249-56.

[126] Klibanov AL, Shevchenko TI, Raju BI, Seip R, Chin CT. Ultrasound-triggered release of materials entrapped in microbubble-liposome constructs: a tool for targeted drug delivery. J Control Release. 2010;148(1) 13-7.

[127] Fan CH, Ting CY, Lin HJ, Wang CH, Liu HL, Yen TC, et al. SPIO-conjugated, doxorubicin-loaded microbubbles for concurrent MRI and focused-ultrasound enhanced brain-tumor drug delivery. Biomaterials. 2013;34(14) 3706-15.

[128] Hernot S, Unnikrishnan S, Du ZM, Shevchenko T, Cosyns B, Broisat A, et al. Nanobody-coupled microbubbles as novel molecular tracer. J Control Release. 2012;158(2) 346-53.

[129] Klibanov AL. Ultrasound molecular imaging with targeted microbubble contrast agents. Journal of nuclear cardiology : official publication of the American Society of Nuclear Cardiology. 2007;14(6) 876-84.

[130] Correas JM, Burns PN, Lai X, Qi X. Infusion versus bolus of an ultrasound contrast agent: in vivo dose-response measurements of BR1. Investigative radiology. 2000;35(1) 72-9.

[131] Albrecht T, Urbank A, Mahler M, Bauer A, Dore CJ, Blomley MJK, et al. Prolongation and optimization of Doppler enhancement with a microbubble US contrast agent by using continuous infusion: Preliminary experience. Radiology. 1998;207(2) 339-47.

[132] Kratzer W, Kachele V, Merkle E, Mason RA, Buchner M, von Tirpitz C, et al. Contrast enhanced power Doppler sonography: Comparison of different administration forms of the ultrasound contrast agent Levovist (R) (vol 172, pg 443, 2000). Rofo-Fortschr Rontg. 2000;172(11) 947-.

[133] Lohmaier S, Ghanem A, Veltmann C, Sommer T, Bruce M, Tiemann K. In vitro and in vivo studies on continuous echo-contrast application strategies using sonovue in a newly developed rotating pump setup. Ultrasound in Medicine and Biology. 2004;30(9) 1145-51. 
[134] Vlasin M, Lukac R, Kauerova Z, Kohout P, Masek J, Bartheldyova E, et al. Specific contrast ultrasound using sterically stabilized microbubbles for early diagnosis of thromboembolic disease in a rabbit model. Canadian journal of veterinary research $=$ Revue canadienne de recherche veterinaire. 2014;78(2) 133-9.

[135] Arita J, Ono Y, Takahashi M, Inoue Y, Takahashi Y, Saiura A. Usefulness of contrastenhanced intraoperative ultrasound in identifying disappearing liver metastases from colorectal carcinoma after chemotherapy. Annals of surgical oncology. 2014;21 Suppl 3 S390-7.

[136] Kiessling F, Fokong S, Bzyl J, Lederle W, Palmowski M, Lammers T. Recent advances in molecular, multimodal and theranostic ultrasound imaging. Advanced drug delivery reviews. 2014;72 15-27.

[137] Alzaraa A, Gravante G, Chung WY, Al-Leswas D, Morgan B, Dennison A, et al. Contrast-enhanced ultrasound in the preoperative, intraoperative and postoperative assessment of liver lesions. Hepatology research : the official journal of the Japan Society of Hepatology. 2013;43(8) 809-19.

[138] de Haas HJ, Narula J, Fuster V. From molecular imaging to pathogenesis and vice versa. Circulation Cardiovascular imaging. 2014;7(4) 581-5.

[139] Kooiman K, Vos HJ, Versluis M, de Jong N. Acoustic behavior of microbubbles and implications for drug delivery. Advanced drug delivery reviews. 2014;72 28-48.

[140] Aryal M, Arvanitis CD, Alexander PM, McDannold N. Ultrasound-mediated bloodbrain barrier disruption for targeted drug delivery in the central nervous system. Advanced drug delivery reviews. 2014;72 94-109.

[141] Burgess A, Hynynen K. Drug delivery across the blood-brain barrier using focused ultrasound. Expert opinion on drug delivery. 2014;11(5) 711-21.

[142] Gao SJ, Zhang Y, Wu JF, Shi WT, Lof J, Vignon F, et al. Improvements in Cerebral Blood Flow and Recanalization Rates With Transcranial Diagnostic Ultrasound and Intravenous Microbubbles After Acute Cerebral Emboli. Investigative radiology. 2014;49(9) 593-600.

[143] de Saint Victor M, Crake C, Coussios CC, Stride E. Properties, characteristics and applications of microbubbles for sonothrombolysis. Expert opinion on drug delivery. 2014;11(2) 187-209.

[144] Kauerova Z, Lukac R, Kohout P., Masek J, Koudelka S, Plockova J, et al. A prototype 'Infucon' device for continuous of microbubbles in vivo. International Journal of Pharmaceutics. 2013;441(1-2) 92-8. 